\title{
A Perishable Inventory System with Postponed Demands and Multiple Server Vacations
}

\author{
R. Jayaraman, B. Sivakumar, and G. Arivarignan \\ Department of Applied Mathematics and Statistics, School of Mathematics, Madurai Kamaraj University, Madurai 625 021, India \\ Correspondence should be addressed to R. Jayaraman, jayarammaths@yahoo.co.in
}

Received 27 March 2011; Revised 7 December 2011; Accepted 16 December 2011

Academic Editor: Agostino Bruzzone

Copyright () 2012 R. Jayaraman et al. This is an open access article distributed under the Creative Commons Attribution License, which permits unrestricted use, distribution, and reproduction in any medium, provided the original work is properly cited.

\begin{abstract}
A mathematical modelling of a continuous review stochastic inventory system with a single server is carried out in this work. We assume that demand time points form a Poisson process. The life time of each item is assumed to have exponential distribution. We assume $(s, S)$ ordering policy to replenish stock with random lead time. The server goes for a vacation of an exponentially distributed duration at the time of stock depletion and may take subsequent vacation depending on the stock position. The customer who arrives during the stock-out period or during the server vacation is offered a choice of joining a pool which is of finite capacity or leaving the system. The demands in the pool are selected one by one by the server only when the inventory level is above $s$, with interval time between any two successive selections distributed as exponential with parameter depending on the number of customers in the pool. The joint probability distribution of the inventory level and the number of customers in the pool is obtained in the steady-state case. Various system performance measures in the steady state are derived, and the long-run total expected cost rate is calculated.
\end{abstract}

\section{Introduction}

In most of the inventory models considered in the literature, the demanded items are directly delivered from the stock (if available). The demands occurring during the stockout period are either lost (lost sales case) or satisfied only after the arrival of ordered items (backlogging). In the latter case, it is assumed that either all (full backlogging) or any prefixed number of demands (partial backlogging) that occurred during the stock-out period are satisfied. The often quoted review articles Nahmias [1] and Raafat [2] and the recent review articles N. H. Shah and Y. K. Shah [3] and Goyal and Giri [4] provide excellent summaries of many of these modelling efforts. For some recent references, see Chakravarthy and Daniel [5], Yadavalli et al. [6], and Kalpakam and Shanthi $[7,8]$.

In the case of continuous review perishable inventory models with random life times for the items, most of the models assume instantaneous supply of order (Lian and Liu [9], Liu and Shi [10], and Kalpakam and Arivarignan [11], and Gürler and Özkaya [12]). When a server is assumed to process the demands in continuous review inventory systems, the concept of server vacation was also taken up in some works. Daniel and Ramanarayanan [13] introduced the concept of server vacation in an inventory model with two servers. In [14], they studied an $(s, S)$ inventory system in which the server takes a rest when the level of the inventory is zero. The demands that occurred during stock-out period were lost. The interoccurrence times between two successive demands, the lead times, and the rest times are assumed to follow general distributions, which are mutually independent. Using renewal and convolution techniques, they obtained the steady-state transition probabilities. However, server vacations in Queueing systems have been widely studied in different contexts in the literature. We refer the readers to Doshi $[15,16]$, Takagi $[17,18]$, and Tian and Zhang [19] for details on various vacations systems and for additional references.

In the context of inventory system, any arriving demands during the server vacation either enter a pool or leave the system. These pooled customers are selected at a later time, preferably after the stock is replenished. This type of inventory problems is called inventory with postponed demands. 
TABLE 1: Optimal values corresponding to various demand rates and the replenishment rates $(\gamma=1.4, \beta=1.3, \alpha=4, p=0.85)$.

\begin{tabular}{|c|c|c|c|c|c|c|c|c|c|c|c|c|c|c|c|}
\hline $\begin{array}{l}\lambda \\
\mu\end{array}$ & & & \multicolumn{3}{|l|}{12} & \multicolumn{3}{|l|}{13} & \multicolumn{3}{|l|}{14} & \multicolumn{2}{|l|}{15} & \multicolumn{2}{|l|}{16} \\
\hline \multirow{2}{*}{4.0} & 9 & 56 & 6 & 10 & 60 & 7 & 11 & 63 & 7 & 12 & 66 & 8 & 13 & 69 & 8 \\
\hline & & 37.484085 & & & 39.299182 & & & 41.092056 & & & 42.865498 & & & 44.620871 & \\
\hline \multirow{2}{*}{4.5} & 9 & 55 & 8 & 9 & 58 & 8 & 10 & 61 & 9 & 11 & 64 & 10 & 12 & 66 & 10 \\
\hline & & 36.545772 & & & 38.252507 & & & 39.930717 & & & 41.591928 & & & 43.235987 & \\
\hline \multirow{2}{*}{5.0} & 8 & 54 & 9 & 9 & 56 & 9 & 10 & 59 & 10 & 10 & 62 & 11 & 11 & 64 & 11 \\
\hline & & 35.729504 & & & 37.353218 & & & 38.95615 & & & 40.53873 & & & 42.08412 & \\
\hline \multirow{2}{*}{5.5} & 8 & 52 & 9 & 8 & 55 & 10 & 9 & 57 & 11 & 10 & 60 & 12 & 11 & 62 & 12 \\
\hline & & 35.077762 & & & 36.606215 & & & 38.119508 & & & 39.621105 & & & 41.11143 & \\
\hline \multirow{2}{*}{6.0} & 7 & 51 & 10 & 8 & 54 & 11 & 9 & 56 & 12 & 9 & 58 & 12 & 10 & 61 & 13 \\
\hline & & 34.470629 & & & 35.957242 & & & 37.433969 & & & 38.854201 & & & 40.262687 & \\
\hline
\end{tabular}

TABLE 2: Optimal values corresponding to various demand rates and perishable rate $(\beta=1.3, \alpha=4, \mu=4, p=0.85)$.

\begin{tabular}{|c|c|c|c|c|c|c|c|c|c|c|c|c|c|c|c|}
\hline$\lambda$ & & 10 & & & 11 & & & 12 & & & 13 & & & 14 & \\
\hline$\gamma$ & & & & & & & & & & & & & & & \\
\hline 1325 & 7 & 50 & 6 & 8 & 54 & 7 & 9 & 57 & 8 & 10 & 60 & 8 & 11 & 60 & 7 \\
\hline $1.5<J$ & & 32.654327 & & & 34.455224 & & & 36.227262 & & & 37.973244 & & & 39.725222 & \\
\hline 1350 & 7 & 50 & 6 & 8 & 53 & 6 & 9 & 57 & 7 & 10 & 60 & 8 & 11 & 60 & 7 \\
\hline 1.530 & & 33.0285 & & & 34.852712 & & & 36.647164 & & & 38.416969 & & & 40.190166 & \\
\hline 1375 & 7 & 50 & 5 & 8 & 53 & 6 & 9 & 57 & 7 & 10 & 60 & 7 & 11 & 60 & 6 \\
\hline נינד & & 33.401547 & & & 35.24819 & & & 37.066484 & & & 38.858742 & & & 40.654683 & \\
\hline 1400 & 7 & 50 & 5 & 8 & 53 & 6 & 9 & 56 & 6 & 10 & 60 & 7 & 11 & 60 & 6 \\
\hline & & 33.771515 & & & 35.643133 & & & 37.484085 & & & 39.299182 & & & 41.115929 & \\
\hline 1425 & 7 & 50 & 5 & 8 & 53 & 5 & 9 & 56 & 6 & 10 & 59 & 6 & 11 & 60 & 6 \\
\hline & & 34.140776 & & & 36.035305 & & & 37.900166 & & & 39.738532 & & & 41.576681 & \\
\hline
\end{tabular}

The concept of postponed demands in the inventory model has been introduced by Berman et al. [20]. They assumed that both demand and service rates are deterministic. Krishnamoorthy and Islam [21] considered an inventory system with Poisson demand, exponential lead time and the pooled customers are selected according to an exponentially distributed time lag. Sivakumar and Arivarignan [22] considered an inventory model in which the demand occurs according to a Markovian arrival process, lead time has a phase type distribution, life time for the items in the stock has exponential distribution, and the pooled customers are selected exponentially. Manuel et al. [23] dealt with an inventory system in which the positive and negative demands occur according to an independent Markovian arrival processes, the lead time of the reorder, the life time of the items, the interselection time of customers from the pool and the reneging time points of the customers in the pool are independent, and each has the exponential distribution. In all the above models, the authors assumed that the pool size is finite. Sivakumar and Arivarignan [24] considered an inventory system with independent Markovian arrival processes for positive and negative demands, exponential lead time for the reorders, exponential life times for each item in the stock, and the pool size is infinite.

In earlier works listed above, the server is assumed to be available even during the stock-out periods. In this work, we have introduced the concept of server vacation. It may be noted that this model also includes situations in which the server may not be availing vacation, but the server may attend some other work during the stock-out period and may return at random times to the inventory system. If the stock is still not replenished, the server goes for another vacation again or to attend the other work. Employees who are assigned extra work on additional remuneration basis can also be thought of such servers.

In this paper, we consider a continuous review perishable inventory system. The unit demands occur according to a Poisson process. The $(s, S)$ ordering policy is implemented to replenish stock. When the inventory is completely depleted, the server is assumed to go on a vacation of random duration. At the end of vacation, if the stock is not replenished, the server again starts his/her vacation. The demands that occurred during stock-out period are offered postponement of their demand after the stock is replenished. Those demands that accept the postponement joins a pool of finite capacity. We assume that fraction $p$ of demands join the pool and the rest leave the system. The pooled demands are selected once the stock is replenished, and the server resumes service. But the selection from the pool will be stopped if the inventory falls below the reorder level, $s$.

The rest of the paper is organized as follows. In Section 2, we describe the mathematical model of the problem under 
TAвLE 3: Optimal values corresponding to various demand rates and the vacation time rates $(\gamma=1.4, \alpha=4, \mu=4, p=0.85)$.

\begin{tabular}{|c|c|c|c|c|c|c|c|c|c|c|c|c|c|c|c|}
\hline \multirow{2}{*}{$\begin{array}{l}\lambda \\
\beta\end{array}$} & \multicolumn{3}{|c|}{12} & \multicolumn{3}{|c|}{13} & \multicolumn{3}{|c|}{14} & \multicolumn{3}{|c|}{15} & \multicolumn{3}{|c|}{16} \\
\hline & & & & & & & & & & & & & & & \\
\hline \multirow{2}{*}{1.30} & 9 & 57 & 6 & 10 & 60 & 7 & 11 & 63 & 7 & 12 & 66 & 8 & 13 & 69 & 8 \\
\hline & & 37.484085 & & & 39.299182 & & & 41.092056 & & & 42.865498 & & & 44.620871 & \\
\hline \multirow{2}{*}{1.35} & 9 & 57 & 8 & 10 & 60 & 9 & 11 & 63 & 9 & 12 & 66 & 10 & 13 & 69 & 10 \\
\hline & & 37.167995 & & & 38.963248 & & & 40.734627 & & & 42.487454 & & & 44.221881 & \\
\hline \multirow{2}{*}{1.40} & 9 & 57 & 9 & 10 & 60 & 10 & 11 & 63 & 11 & 12 & 66 & 11 & 13 & 69 & 12 \\
\hline & & 36.861364 & & & 38.635679 & & & 40.388064 & & & 42.120116 & & & 43.834419 & \\
\hline \multirow{2}{*}{1.45} & 9 & 56 & 10 & 9 & 59 & 11 & 10 & 62 & 12 & 11 & 66 & 13 & 12 & 69 & 14 \\
\hline & & 36.566275 & & & 38.316645 & & & 40.041077 & & & 41.748416 & & & 43.440092 & \\
\hline \multirow{2}{*}{1.50} & 8 & 56 & 11 & 9 & 59 & 12 & 10 & 62 & 13 & 11 & 65 & 14 & 12 & 68 & 15 \\
\hline & & 36.260058 & & & 37.985767 & & & 39.692538 & & & 41.381935 & & & 43.055515 & \\
\hline
\end{tabular}

TABLE 4: Effect of primary and postponed demand rates on the optimal values $(\gamma=1.4, \beta=1.3, \mu=4, p=0.85)$.

\begin{tabular}{|c|c|c|c|c|c|c|c|c|c|c|c|c|c|c|c|}
\hline$\lambda$ & & 10 & & & 11 & & & 12 & & & 13 & & & 14 & \\
\hline$\alpha$ & & & & & & & & & & & & & & & \\
\hline 4.0 & 7 & 50 & 5 & 8 & 53 & 6 & 9 & 56 & 6 & 10 & 60 & 7 & 11 & 63 & 7 \\
\hline 4.0 & & 33.771515 & & & 35.643133 & & & 37.484085 & & & 39.299182 & & & 41.092056 & \\
\hline 45 & 7 & 50 & 6 & 8 & 54 & 7 & 9 & 57 & 8 & 10 & 60 & 8 & 11 & 63 & 9 \\
\hline $4 . J$ & & 33.690772 & & & 35.556042 & & & 37.392308 & & & 39.202171 & & & 40.990676 & \\
\hline 50 & 7 & 51 & 7 & 8 & 54 & 8 & 9 & 57 & 8 & 10 & 60 & 9 & 11 & 63 & 10 \\
\hline 5.0 & & 33.618558 & & & 35.478749 & & & 37.310908 & & & 39.116144 & & & 40.900342 & \\
\hline 55 & 7 & 51 & 8 & 8 & 54 & 8 & 9 & 57 & 9 & 10 & 60 & 10 & 11 & 63 & 10 \\
\hline 3.0 & & 33.555137 & & & 35.41211 & & & 37.2389 & & & 39.041211 & & & 40.821941 & \\
\hline 60 & 7 & 51 & 8 & 8 & 54 & 9 & 9 & 57 & 10 & 10 & 60 & 10 & 11 & 63 & 11 \\
\hline & & 33.499545 & & & 35.352615 & & & 37.177226 & & & 38.976372 & & & 40.752532 & \\
\hline
\end{tabular}

study. The steady-state analysis of the model is presented in Section 3, and some key system performance measures are derived in Section 4. In Section 5, we calculate the total expected cost rate, and, in the final section, we perform sensitivity analyses on the total expected cost rate in terms of numerical illustrations.

\section{Problem Formulation}

We consider an inventory system with a maximum stock of $S$ unit items. The items are assumed to have identical and independent exponential life-time distributions with parameter $\gamma(>0)$. When an item perishes, it is removed from the stock. Another process which removes items from the stock is the demand process. The demand is for single item, and the demand occurrences form a Poisson process with rate $\lambda(>0)$. A server processes the unit demands. The stock is replenished by the server by implementing $(s, S)$ ordering policy, where the lead time is assumed to have independent exponential distribution with parameter $\mu(>0)$. If the stock is depleted before the supply of reorder, the server goes on a vacation duration which has exponential distribution with parameter $\beta(>0)$. At the end of a vacation period, if the stock is still empty, the server takes another vacation. The demands that occur during stock-out period are offered postponement at a later time. It is assumed that the demands accept this

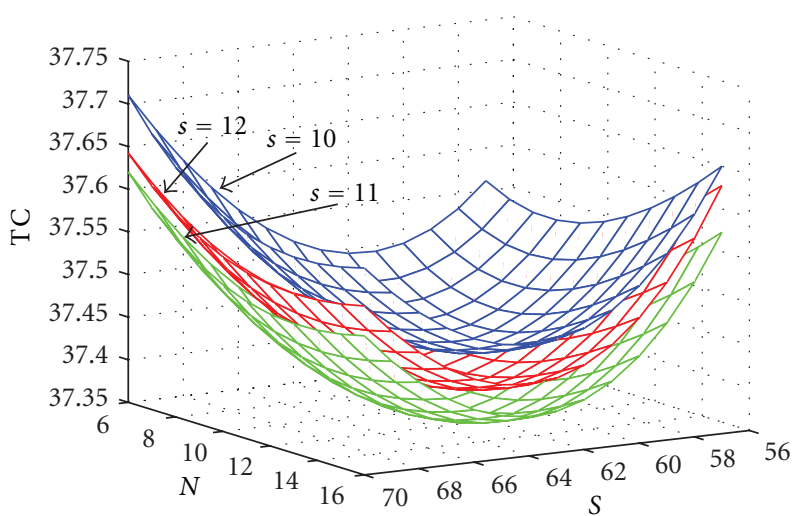

FIGURE 1: A three-dimensional plot of the cost function. $\lambda=14$, $\mu=4, \gamma=1.2, \beta=1.3, \alpha_{i}=i * \alpha, i=1,2, \ldots, N, \alpha=4$, $p=0.85, c_{h}=0.3, c_{s}=15, c_{\mathrm{pr}}=0.3, c_{\mathrm{sh}}=5, c_{p}=3$.

postponement or leave the system according to a Bernoulli distribution with probability $p$. The demand which accepts an offer of postponement may join a pool of finite capacity fixed as $N$.

The demands from the pool are selected if the server is on service and the inventory level is above $s$ such that the interval time between successive selections is exponentially 
TABLE 5: Optimal values corresponding to various perishable rates and the replenishment rates $(\lambda=14, \beta=1.3, \alpha=4, p=0.85)$.

\begin{tabular}{|c|c|c|c|c|c|c|c|c|c|c|c|c|c|c|c|}
\hline $\begin{array}{l}\mu \\
\gamma \\
\end{array}$ & \multicolumn{3}{|c|}{4.0} & \multicolumn{3}{|c|}{4.5} & \multicolumn{3}{|c|}{5.0} & \multicolumn{3}{|c|}{5.5} & \multicolumn{3}{|c|}{6.0} \\
\hline \multirow{2}{*}{1.30} & 11 & 63 & 9 & 10 & 61 & 11 & 10 & 59 & 12 & 9 & 57 & 13 & 9 & 56 & 13 \\
\hline & & 39.231174 & & & 38.117777 & & & 37.192369 & & & 36.39762 & & & 35.754118 & \\
\hline \multirow{2}{*}{1.35} & 11 & 63 & 8 & 10 & 61 & 10 & 10 & 59 & 11 & 9 & 57 & 12 & 9 & 56 & 12 \\
\hline & & 40.164239 & & & 39.025748 & & & 38.075018 & & & 37.259064 & & & 36.594523 & \\
\hline \multirow{2}{*}{1.40} & 11 & 63 & 7 & 10 & 61 & 9 & 10 & 59 & 10 & 9 & 57 & 11 & 9 & 56 & 12 \\
\hline & & 41.092056 & & & 39.930717 & & & 38.95615 & & & 38.119508 & & & 37.433969 & \\
\hline \multirow{2}{*}{1.45} & 11 & 63 & 6 & 10 & 61 & 8 & 10 & 59 & 9 & 9 & 57 & 10 & 9 & 56 & 11 \\
\hline & & 42.013602 & & & 40.832153 & & & 39.835433 & & & 38.978705 & & & 38.272741 & \\
\hline \multirow{2}{*}{1.50} & 11 & 62 & 5 & 10 & 61 & 7 & 10 & 59 & 9 & 9 & 56 & 10 & 9 & 56 & 10 \\
\hline & & 42.926105 & & & 41.7295 & & & 40.711703 & & & 39.83621 & & & 39.110758 & \\
\hline
\end{tabular}

TABLE 6: Influence of the postponed demand rate and replenishment rate on the optimal values $(\lambda=14, \gamma=1.4, \beta=1.3, p=0.85)$.

\begin{tabular}{|c|c|c|c|c|c|c|c|c|c|c|c|c|c|c|c|}
\hline $\begin{array}{l}\mu \\
\alpha \\
\end{array}$ & \multicolumn{3}{|c|}{4.0} & \multicolumn{3}{|c|}{4.5} & \multicolumn{3}{|c|}{5.0} & \multicolumn{3}{|c|}{5.5} & \multicolumn{3}{|c|}{6.0} \\
\hline \multirow{2}{*}{3.50} & 11 & 62 & 4 & 10 & 60 & 7 & 10 & 59 & 9 & 9 & 57 & 9 & 9 & 56 & 10 \\
\hline & & 41.194355 & & & 40.049416 & & & 39.065602 & & & 38.230701 & & & 37.531787 & \\
\hline \multirow{2}{*}{3.75} & 11 & 62 & 6 & 10 & 61 & 8 & 10 & 59 & 9 & 9 & 57 & 10 & 9 & 56 & 11 \\
\hline & & 41.145131 & & & 39.988997 & & & 39.009046 & & & 38.17254 & & & 37.480279 & \\
\hline \multirow{2}{*}{4.00} & 11 & 63 & 7 & 10 & 61 & 9 & 10 & 59 & 10 & 9 & 57 & 11 & 9 & 56 & 12 \\
\hline & & 41.092056 & & & 39.930717 & & & 38.95615 & & & 38.119508 & & & 37.433969 & \\
\hline \multirow{2}{*}{4.25} & 11 & 63 & 8 & 10 & 61 & 10 & 10 & 59 & 11 & 9 & 57 & 11 & 9 & 56 & 12 \\
\hline & & 41.039824 & & & 39.876714 & & & 38.908033 & & & 38.071643 & & & 37.391719 & \\
\hline \multirow{2}{*}{4.50} & 11 & 63 & 9 & 10 & 61 & 10 & 10 & 59 & 11 & 9 & 57 & 12 & 8 & 56 & 13 \\
\hline & & 40.990676 & & & 39.826149 & & & 38.863594 & & & 38.027026 & & & 37.349602 & \\
\hline
\end{tabular}

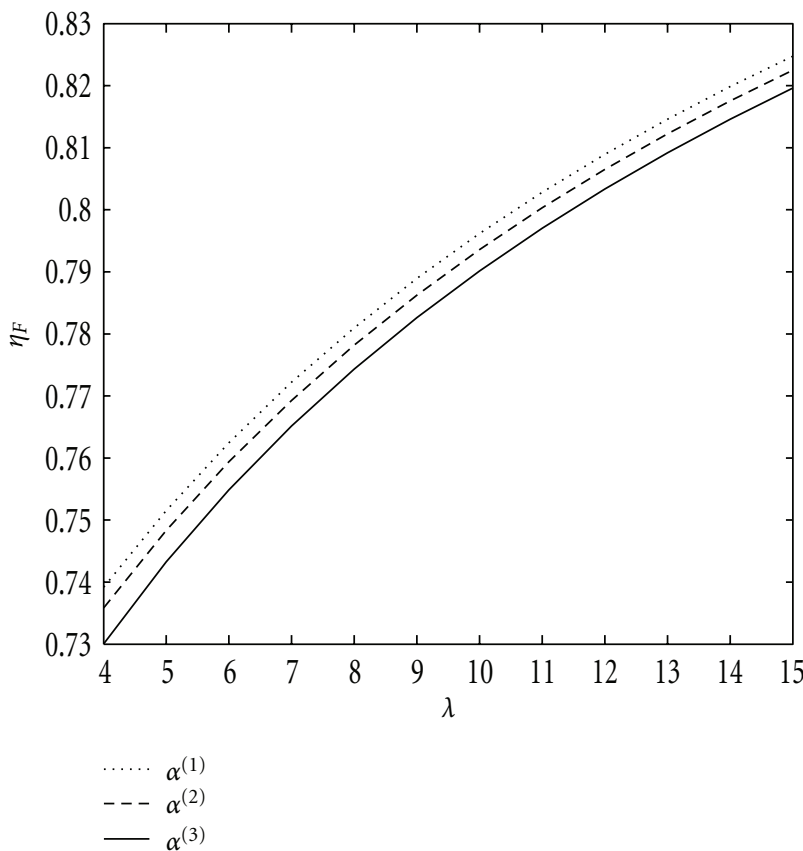

FIGURE 2: Fraction of time server is on vacation- $\lambda$ versus $\alpha^{(n)}(S=$ 90, $s=15, N=25, \beta=1.4, \gamma=4, \mu=0.8, p=0.75$.)

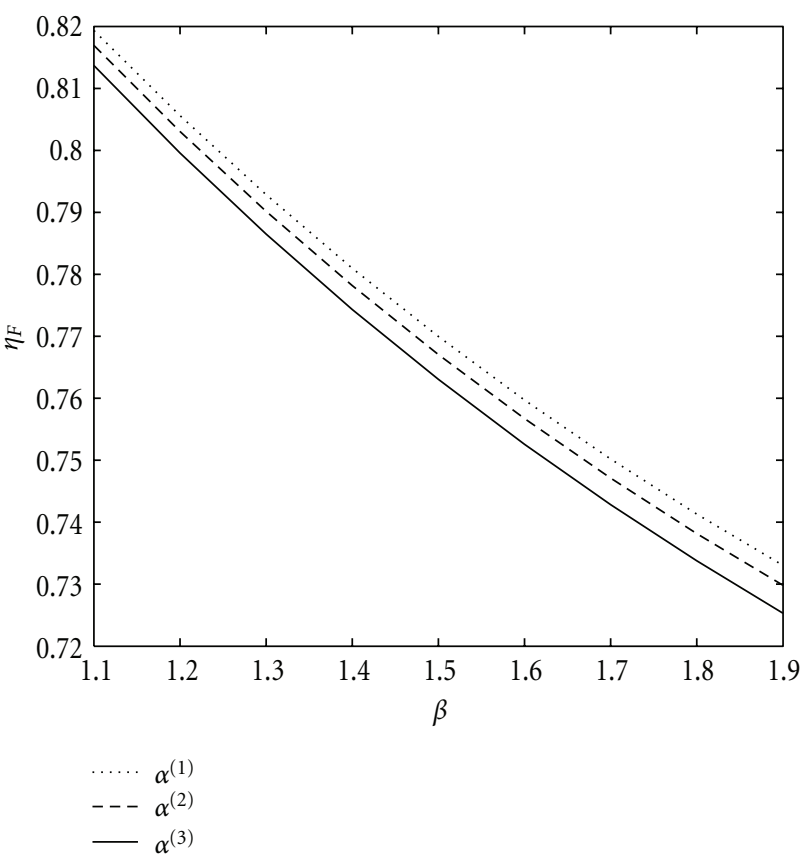

FIgURE 3: Fraction of time server is on vacation- $\beta$ versus $\alpha^{(n)} \quad(S=$ 90, $s=15, N=25, \lambda=8, \gamma=4, \mu=0.8, p=0.75$.) 
TABLE 7: Influence of the vacation time rate and the replenishment rate on the optimal values $(\lambda=14, \gamma=1.4, \alpha=4, p=0.85)$.

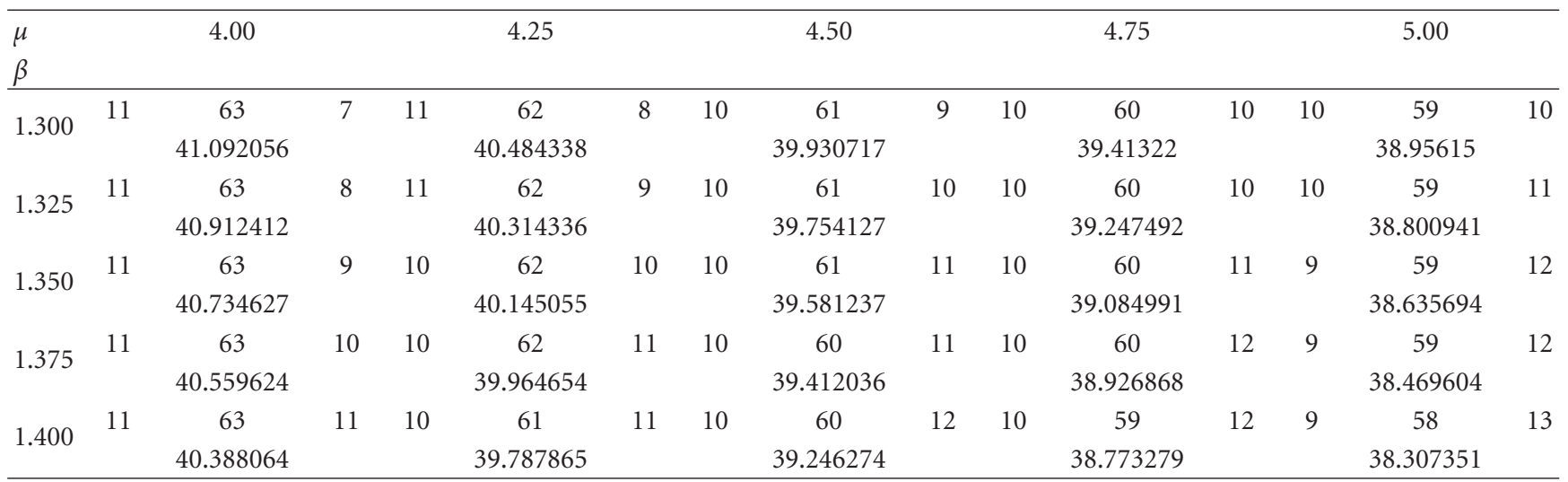

TABLE 8: Optimal values corresponding to various perishable rates and vacation time rates $(\lambda=14, \alpha=4, \mu=4, p=0.85)$.

\begin{tabular}{|c|c|c|c|c|c|c|c|c|c|c|c|c|c|c|c|}
\hline$\beta$ & & 1.30 & & & 1.35 & & & 1.40 & & & 1.45 & & & 1.50 & \\
\hline \multicolumn{16}{|l|}{$\gamma$} \\
\hline \multirow{2}{*}{1.30} & 11 & 63 & 9 & 11 & 63 & 11 & 11 & 63 & 12 & 10 & 62 & 14 & 10 & 62 & 15 \\
\hline & & 39.231174 & & & 38.886899 & & & 38.556819 & & & 38.226251 & & & 37.897017 & \\
\hline \multirow{2}{*}{1.35} & 11 & 63 & 8 & 11 & 63 & 10 & 11 & 63 & 11 & 10 & 62 & 13 & 10 & 62 & 14 \\
\hline & & 40.164239 & & & 39.812693 & & & 39.474237 & & & 39.134974 & & & 38.795693 & \\
\hline \multirow{2}{*}{1.40} & 11 & 63 & 7 & 11 & 63 & 9 & 11 & 63 & 11 & 10 & 62 & 12 & 10 & 62 & 13 \\
\hline & & 41.092056 & & & 40.734627 & & & 40.388064 & & & 40.041077 & & & 39.692538 & \\
\hline \multirow{2}{*}{1.45} & 11 & 63 & 6 & 11 & 63 & 8 & 11 & 63 & 10 & 10 & 62 & 11 & 10 & 62 & 12 \\
\hline & & 42.013602 & & & 41.652036 & & & 41.298322 & & & 40.944106 & & & 40.587172 & \\
\hline \multirow{2}{*}{1.50} & 11 & 62 & 5 & 11 & 63 & 7 & 11 & 63 & 9 & 10 & 63 & 11 & 10 & 62 & 12 \\
\hline & & 42.926105 & & & 42.564207 & & & 42.204506 & & & 41.84354 & & & 41.478112 & \\
\hline
\end{tabular}

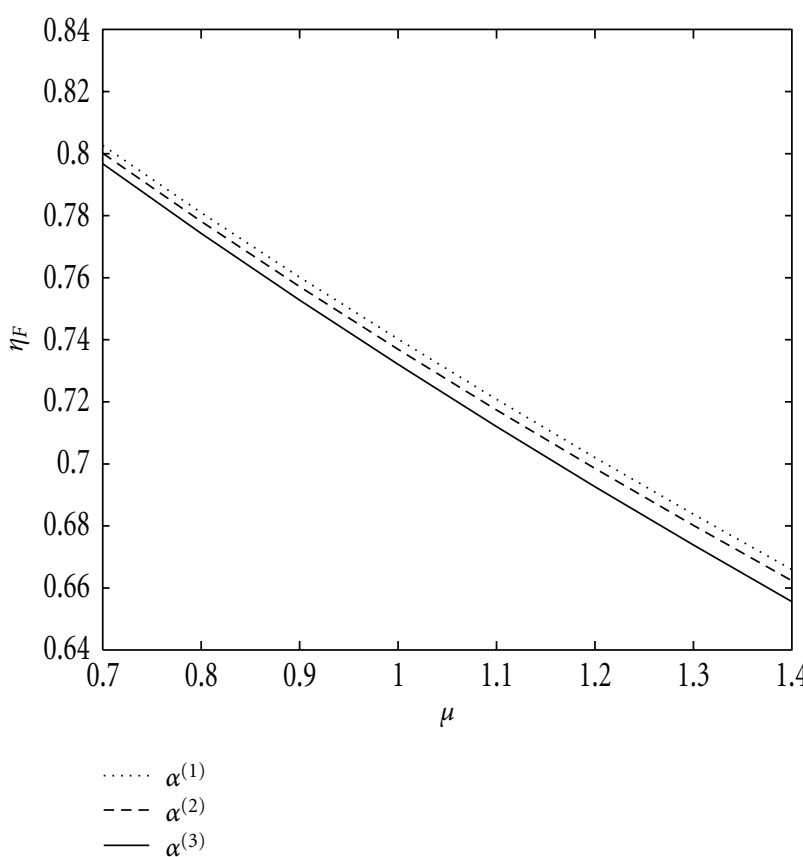

FIGURE 4: Fraction of time server is on vacation- $\mu$ versus $\alpha^{(n)}(S=$ 90, $s=15, N=25, \beta=1.4, \gamma=4, \lambda=8, p=0.75$.)

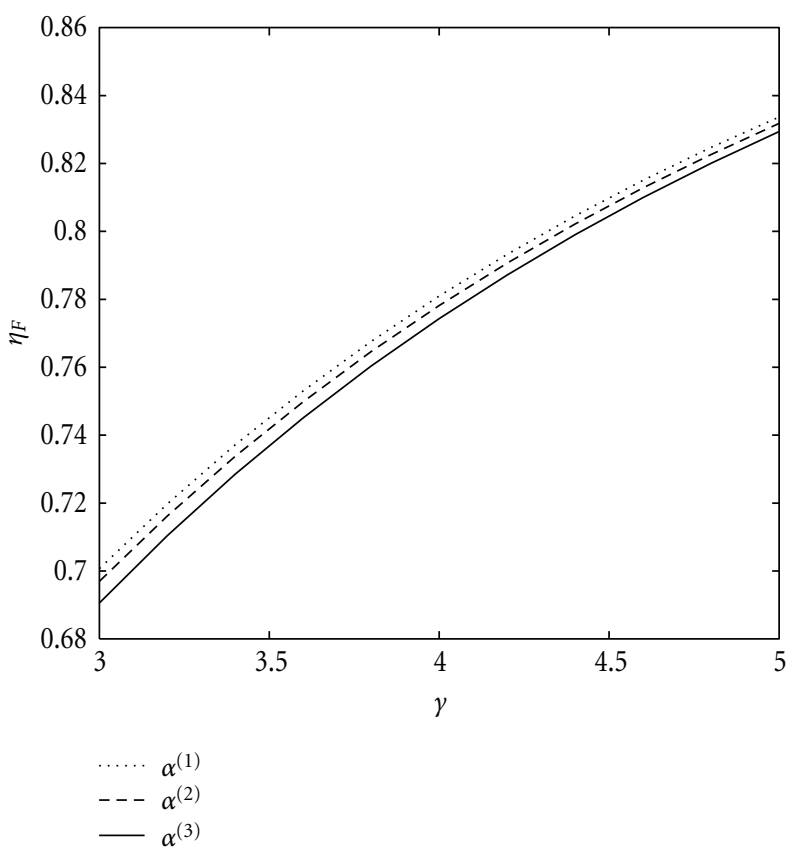

FIGURE 5: Fraction of time server is on vacation- $\gamma$ versus $\alpha^{(n)}(S=$ 90, $s=15, N=25, \beta=1.4, \lambda=8, \mu=0.8, p=0.75$.) 
TABLE 9: Influence of the postponed demand rate and vacation time rate on the optimal values $(\lambda=14, \gamma=1.4, \mu=4, p=0.85)$.

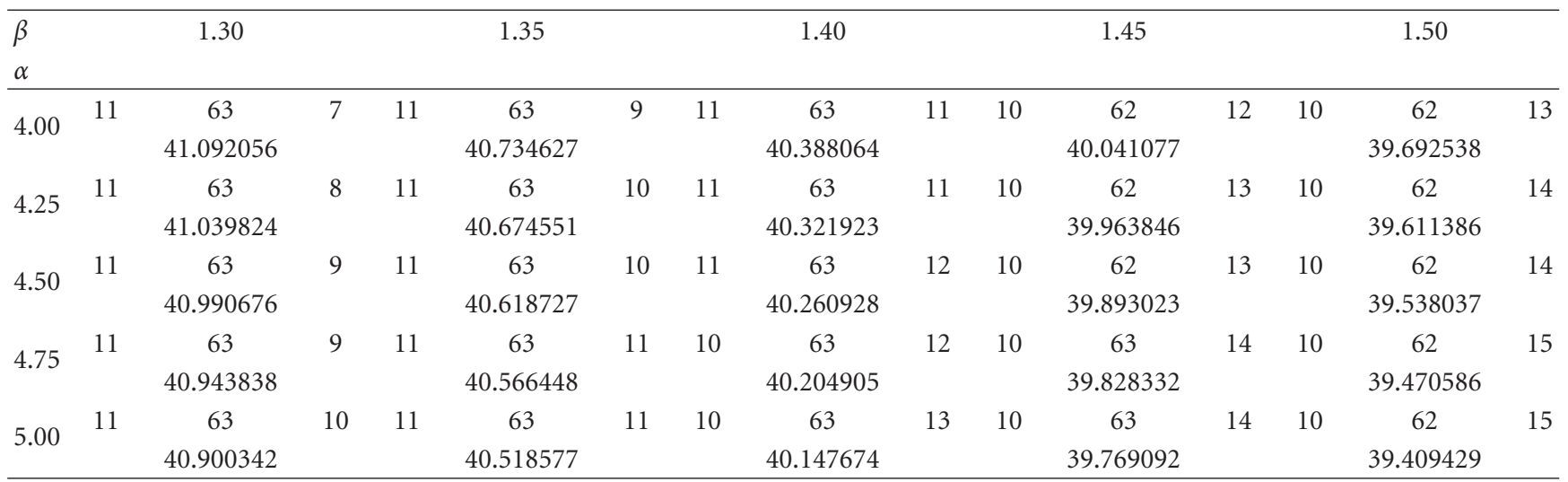

TABLe 10: Optimal values corresponding to various perishable rates and postponed demand rates $(\lambda=14, \beta=1.3, \mu=4, p=0.85)$.

\begin{tabular}{|c|c|c|c|c|c|c|c|c|c|c|c|c|c|c|c|}
\hline \multirow{2}{*}{$\begin{array}{l}\alpha \\
\gamma\end{array}$} & \multicolumn{3}{|c|}{4.00} & \multicolumn{3}{|c|}{4.25} & \multicolumn{3}{|c|}{4.50} & \multicolumn{3}{|c|}{4.75} & \multicolumn{3}{|c|}{5.00} \\
\hline & & & & & & & & & & & & & & & \\
\hline \multirow{2}{*}{1.0} & 11 & 62 & 15 & 11 & 62 & 16 & 11 & 62 & 16 & 11 & 62 & 17 & 11 & 62 & 17 \\
\hline & & 33.566483 & & & 33.512828 & & & 33.46372 & & & 33.419215 & & & 33.378523 & \\
\hline \multirow{2}{*}{1.1} & 11 & 62 & 13 & 11 & 62 & 13 & 11 & 63 & 14 & 11 & 63 & 15 & 11 & 63 & 15 \\
\hline & & 35.463386 & & & 35.407926 & & & 35.357122 & & & 35.310631 & & & 35.268167 & \\
\hline \multirow{2}{*}{1.2} & 11 & 63 & 11 & 11 & 63 & 12 & 11 & 63 & 12 & 11 & 63 & 13 & 11 & 63 & 13 \\
\hline & & 37.352776 & & & 37.296972 & & & 37.245085 & & & 37.197543 & & & 37.153723 & \\
\hline \multirow{2}{*}{1.3} & 11 & 63 & 9 & 11 & 63 & 10 & 11 & 63 & 10 & 11 & 63 & 11 & 11 & 63 & 11 \\
\hline & & 39.231174 & & & 39.176278 & & & 39.125134 & & & 39.077093 & & & 39.033214 & \\
\hline \multirow{2}{*}{1.4} & 11 & 63 & 7 & 11 & 63 & 8 & 11 & 63 & 9 & 11 & 63 & 9 & 11 & 63 & 10 \\
\hline & & 41.092056 & & & 41.039824 & & & 40.990676 & & & 40.943838 & & & 40.900342 & \\
\hline
\end{tabular}

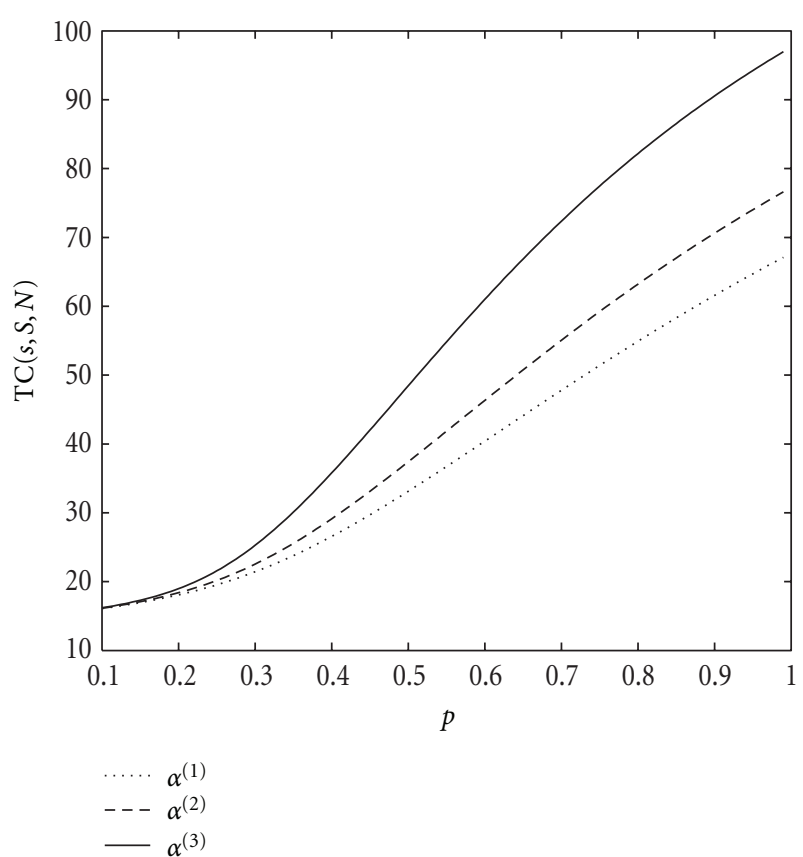

FIGURE 6: The total expected cost rate- $p$ versus $\alpha^{(n)}(S=90, s=$ $15, N=25, \lambda=8, \beta=1.4, \gamma=0.7, \lambda=8$.)

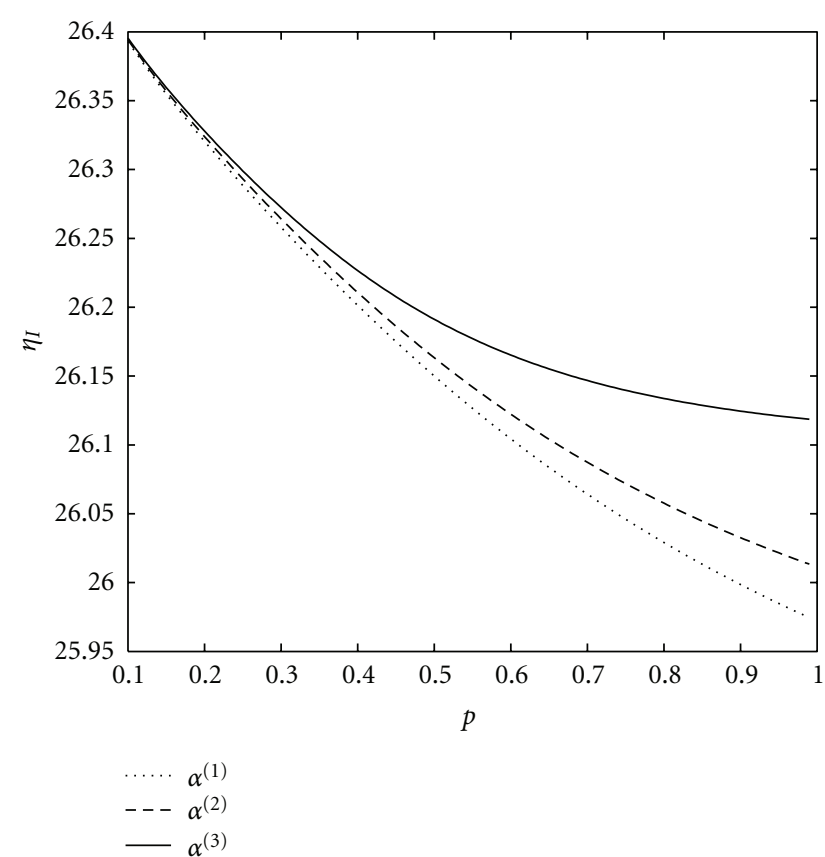

FIGURE 7: Expected Inventory Level- $p$ versus $\alpha^{(n)}(S=90, s=$ $15, N=25, \lambda=8, \beta=1.4, \gamma=0.7, \mu=0.8$.) 
TABLe 11: Variation in optimal values for different values of $c_{h}$ and $c_{p}\left(c_{s}=15, c_{\mathrm{sh}}=5, c_{\mathrm{pr}}=0.3\right)$.

\begin{tabular}{|c|c|c|c|c|c|c|c|c|c|c|c|c|c|c|c|}
\hline \multirow{2}{*}{$\begin{array}{l}c_{h} \\
c_{p} \\
\end{array}$} & \multicolumn{3}{|c|}{0.20} & \multicolumn{3}{|c|}{0.25} & \multicolumn{3}{|c|}{0.30} & \multicolumn{3}{|c|}{0.35} & \multicolumn{3}{|c|}{0.40} \\
\hline & & & & & & & & & & & & & & & \\
\hline \multirow{2}{*}{2.2} & 11 & 69 & 24 & 10 & 66 & 22 & 10 & 63 & 21 & 10 & 61 & 21 & 10 & 59 & 20 \\
\hline & & 37.185505 & & & 38.452295 & & & 39.665423 & & & 40.837851 & & & 41.974311 & \\
\hline \multirow{2}{*}{2.4} & 11 & 69 & 19 & 11 & 66 & 18 & 10 & 64 & 18 & 10 & 61 & 17 & 10 & 59 & 16 \\
\hline & & 37.599174 & & & 38.884988 & & & 40.125458 & & & 41.301575 & & & 42.440661 & \\
\hline \multirow{2}{*}{2.6} & 11 & 69 & 16 & 11 & 66 & 15 & 11 & 64 & 14 & 11 & 61 & 13 & 10 & 59 & 13 \\
\hline & & 37.972831 & & & 39.260922 & & & 40.503633 & & & 41.705424 & & & 42.851175 & \\
\hline \multirow{2}{*}{2.8} & 11 & 69 & 12 & 11 & 66 & 12 & 11 & 63 & 11 & 11 & 61 & 10 & 11 & 59 & 9 \\
\hline & & 38.303582 & & & 39.590386 & & & 40.831047 & & & 42.028107 & & & 43.188119 & \\
\hline \multirow{2}{*}{3.0} & 12 & 69 & 9 & 11 & 66 & 8 & 11 & 63 & 7 & 11 & 60 & 6 & 11 & 58 & 5 \\
\hline & & 38.563014 & & & 39.86128 & & & 41.092056 & & & 42.278538 & & & 43.424151 & \\
\hline
\end{tabular}

TABLE 12: Optimal values corresponding to various holding and perishable costs $\left(c_{s}=15, c_{\mathrm{sh}}=5, c_{p}=3\right)$.

\begin{tabular}{|c|c|c|c|c|c|c|c|c|c|c|c|c|c|c|c|}
\hline \multirow{2}{*}{$\begin{array}{l}c_{h} \\
c_{\mathrm{pr}} \\
\end{array}$} & \multicolumn{3}{|c|}{0.20} & \multicolumn{3}{|c|}{0.25} & \multicolumn{3}{|c|}{0.30} & \multicolumn{3}{|c|}{0.35} & \multicolumn{3}{|c|}{0.40} \\
\hline & & & & & & & & & & & & & & & \\
\hline \multirow{2}{*}{0.250} & 12 & 74 & 10 & 12 & 70 & 9 & 12 & 67 & 8 & 11 & 64 & 8 & 11 & 61 & 6 \\
\hline & & 36.642829 & & & 38.025913 & & & 39.353537 & & & 40.605434 & & & 41.809696 & \\
\hline \multirow{2}{*}{0.275} & 12 & 71 & 10 & 12 & 68 & 9 & 11 & 65 & 8 & 11 & 62 & 7 & 11 & 60 & 6 \\
\hline & & 37.617513 & & & 38.960668 & & & 40.235119 & & & 41.452256 & & & 42.627321 & \\
\hline \multirow{2}{*}{0.300} & 12 & 69 & 9 & 11 & 66 & 8 & 11 & 63 & 7 & 11 & 60 & 6 & 11 & 58 & 5 \\
\hline & & 38.563014 & & & 39.86128 & & & 41.092056 & & & 42.278538 & & & 43.424151 & \\
\hline \multirow{2}{*}{0.325} & 11 & 66 & 8 & 11 & 63 & 7 & 11 & 61 & 6 & 11 & 58 & 5 & 11 & 56 & 4 \\
\hline & & 39.482691 & & & 40.72827 & & & 41.927648 & & & 43.085434 & & & 44.202381 & \\
\hline \multirow{2}{*}{0.350} & 11 & 65 & 8 & 11 & 62 & 7 & 11 & 59 & 6 & 11 & 57 & 5 & 10 & 54 & 3 \\
\hline & & 40.359663 & & & 41.571843 & & & 42.741939 & & & 43.871344 & & & 44.952053 & \\
\hline
\end{tabular}

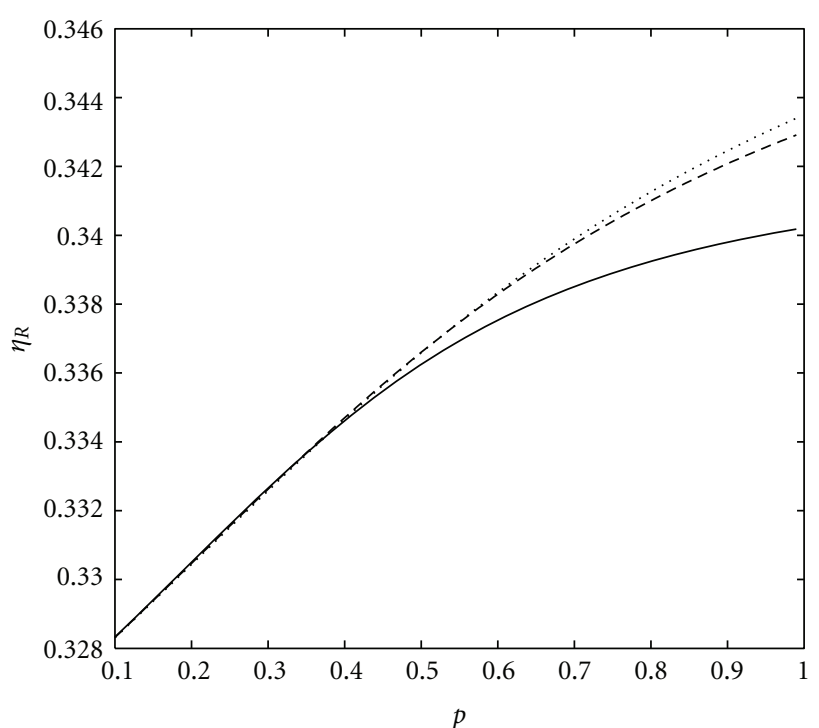

$$
\begin{gathered}
\ldots \alpha^{(1)} \\
---\alpha^{(2)} \\
-\quad \alpha^{(3)}
\end{gathered}
$$

Figure 8: Expected reorder rate- $p$ versus $\alpha^{(n)}(S=90, s=$ $15, N=25, \lambda=8, \beta=1.4, \gamma=0.7, \mu=0.8$.)

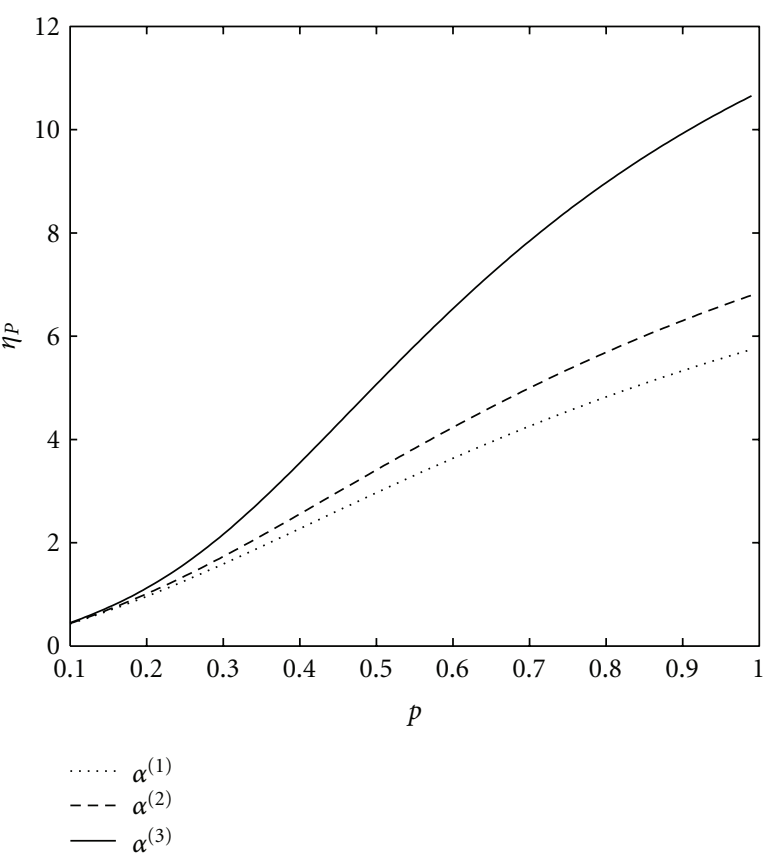

Figure 9: Expected number of customers in the pool- $p$ versus $\alpha^{(n)}(S=90, s=15, N=25, \lambda=8, \beta=1.4, \gamma=0.7, \mu=0.8$. 
TABLE 13: Optimal values corresponding to various setup and holding costs $\left(c_{\mathrm{sh}}=5, c_{p}=3, c_{\mathrm{pr}}=0.3\right)$.

\begin{tabular}{|c|c|c|c|c|c|c|c|c|c|c|c|c|c|c|c|}
\hline \multirow{2}{*}{$\begin{array}{l}c_{h} \\
c_{s}\end{array}$} & \multicolumn{3}{|c|}{0.20} & \multicolumn{3}{|c|}{0.25} & \multicolumn{3}{|c|}{0.30} & \multicolumn{3}{|c|}{0.35} & \multicolumn{3}{|c|}{0.40} \\
\hline & & & & & & & & & & & & & & & \\
\hline \multirow{2}{*}{13} & 12 & 66 & 8 & 12 & 63 & 7 & 12 & 61 & 7 & 11 & 58 & 6 & 11 & 55 & 4 \\
\hline & & 36.775469 & & & 38.037617 & & & 39.253444 & & & 40.42214 & & & 41.527663 & \\
\hline \multirow{2}{*}{14} & 12 & 68 & 9 & 12 & 65 & 8 & 11 & 62 & 7 & 11 & 59 & 6 & 11 & 57 & 5 \\
\hline & & 37.674734 & & & 38.959441 & & & 40.189174 & & & 41.355166 & & & 42.481256 & \\
\hline \multirow{2}{*}{15} & 12 & 69 & 9 & 11 & 66 & 8 & 11 & 63 & 7 & 11 & 60 & 6 & 11 & 58 & 5 \\
\hline & & 38.563014 & & & 39.86128 & & & 41.092056 & & & 42.278538 & & & 43.424151 & \\
\hline \multirow{2}{*}{16} & 11 & 70 & 9 & 11 & 67 & 8 & 11 & 64 & 7 & 11 & 61 & 6 & 10 & 58 & 5 \\
\hline & & 39.433976 & & & 40.734783 & & & 41.986075 & & & 43.19263 & & & 44.356396 & \\
\hline \multirow{2}{*}{17} & 11 & 72 & 9 & 11 & 68 & 9 & 11 & 65 & 8 & 10 & 62 & 7 & 10 & 60 & 6 \\
\hline & & 40.282373 & & & 41.600143 & & & 42.871236 & & & 44.094213 & & & 45.249004 & \\
\hline
\end{tabular}

TABLE 14: Variation in optimal values for different values of $c_{h}$ and $c_{\mathrm{sh}}\left(c_{s}=15, c_{p}=3, c_{\mathrm{pr}}=0.3\right)$.

\begin{tabular}{|c|c|c|c|c|c|c|c|c|c|c|c|c|c|c|c|}
\hline \multirow{2}{*}{$\begin{array}{l}c_{h} \\
c_{\text {sh }} \\
\end{array}$} & \multicolumn{3}{|c|}{0.20} & \multicolumn{3}{|c|}{0.25} & \multicolumn{3}{|c|}{0.30} & \multicolumn{3}{|c|}{0.35} & \multicolumn{3}{|c|}{0.40} \\
\hline & & & & & & & & & & & & & & & \\
\hline \multirow{2}{*}{5.00} & 12 & 69 & 9 & 11 & 66 & 8 & 11 & 63 & 7 & 11 & 60 & 6 & 11 & 58 & 5 \\
\hline & & 38.563014 & & & 39.86128 & & & 41.092056 & & & 42.278538 & & & 43.424151 & \\
\hline \multirow{2}{*}{5.25} & 12 & 70 & 12 & 12 & 67 & 11 & 11 & 64 & 10 & 11 & 62 & 10 & 11 & 60 & 9 \\
\hline & & 38.811315 & & & 40.138832 & & & 41.406634 & & & 42.618679 & & & 43.790972 & \\
\hline \multirow{2}{*}{5.50} & 12 & 71 & 14 & 12 & 68 & 13 & 12 & 66 & 13 & 11 & 63 & 12 & 11 & 61 & 11 \\
\hline & & 39.026709 & & & 40.371016 & & & 41.668858 & & & 42.905412 & & & 44.097789 & \\
\hline \multirow{2}{*}{5.75} & 12 & 72 & 16 & 12 & 69 & 16 & 12 & 66 & 15 & 12 & 64 & 14 & 11 & 62 & 14 \\
\hline & & 39.219457 & & & 40.577343 & & & 41.889389 & & & 43.15853 & & & 44.366545 & \\
\hline \multirow{2}{*}{6.00} & 12 & 73 & 19 & 12 & 70 & 18 & 12 & 67 & 17 & 12 & 65 & 16 & 11 & 63 & 16 \\
\hline & & 39.394802 & & & 40.764441 & & & 42.08824 & & & 43.370604 & & & 44.60868 & \\
\hline
\end{tabular}

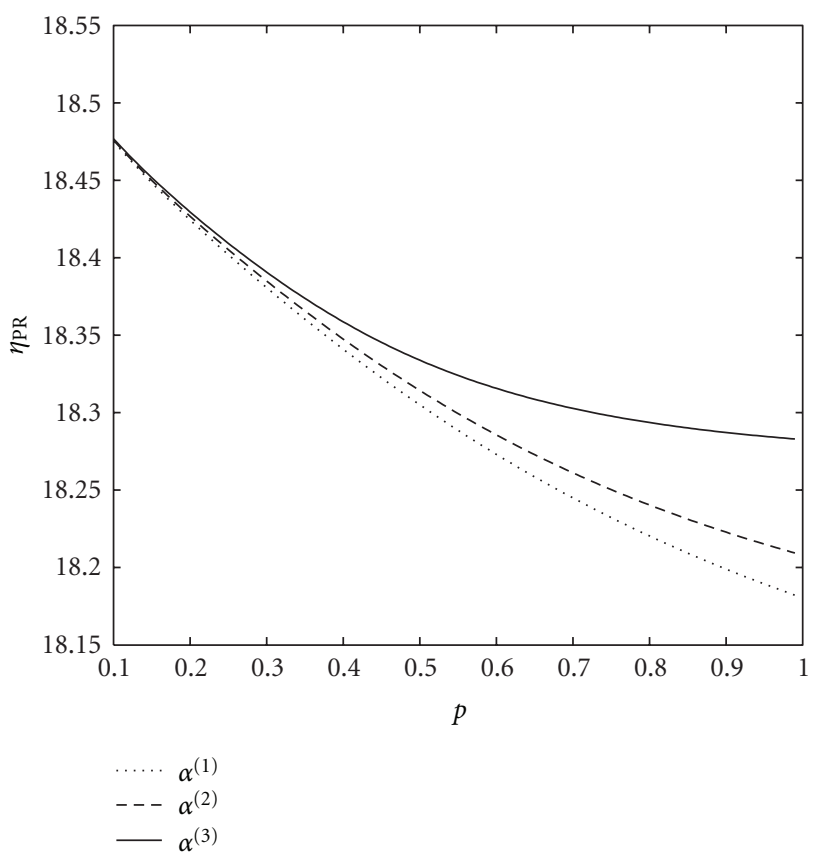

Figure 10: Expected perishable rate- $p$ versus $\alpha^{(n)}(S=90, s=$ $15, N=25, \lambda=8, \beta=1.4, \gamma=0.7, \mu=0.8$.)

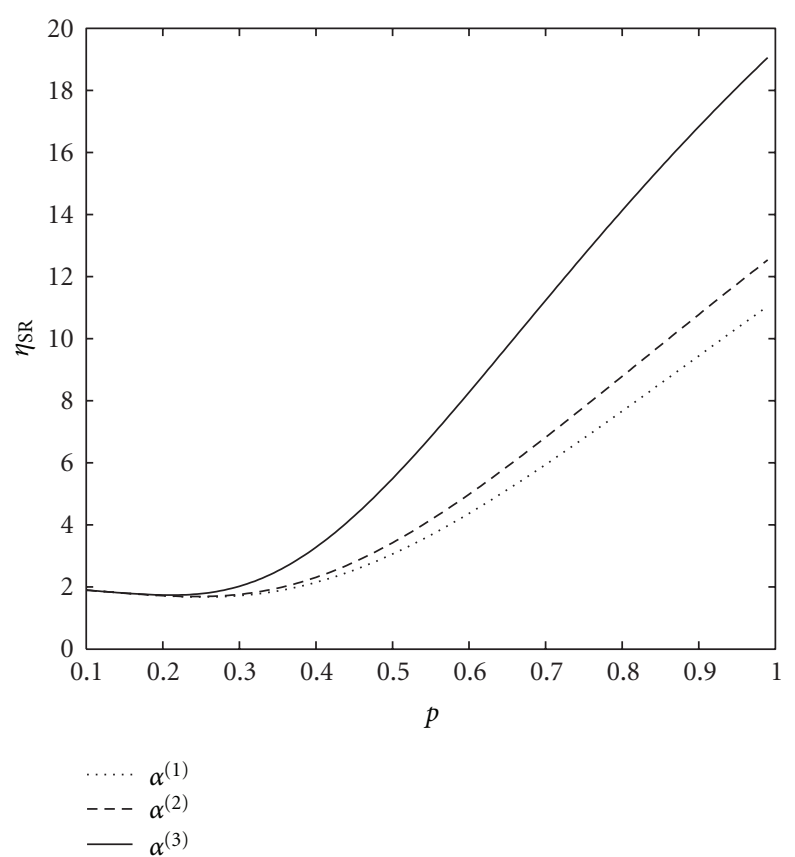

FIgURE 11: Mean loss of demand rate- $p$ versus $\alpha^{(n)}(S=90, s=$ $15, N=25, \lambda=8, \beta=1.4, \gamma=0.7, \mu=0.8$.) 
TABLE 15: Effect of $c_{s}$ and $c_{p}$ on the optimal cost rate $\left(c_{h}=0.3, c_{\mathrm{sh}}=5, c_{\mathrm{pr}}=0.3\right)$.

\begin{tabular}{|c|c|c|c|c|c|c|c|c|c|c|c|c|c|c|c|}
\hline \multirow{2}{*}{$\begin{array}{l}c_{s} \\
c_{p}\end{array}$} & \multicolumn{3}{|c|}{14.0} & \multicolumn{3}{|c|}{14.5} & \multicolumn{3}{|c|}{15.0} & \multicolumn{3}{|c|}{15.5} & \multicolumn{3}{|c|}{16.0} \\
\hline & & & & & & & & & & & & & & & \\
\hline \multirow{2}{*}{2.2} & 10 & 63 & 21 & 10 & 63 & 21 & 10 & 64 & 21 & 10 & 64 & 22 & 10 & 65 & 22 \\
\hline & & 38.786969 & & & 39.226893 & & & 39.665423 & & & 40.100898 & & & 40.535658 & \\
\hline \multirow{2}{*}{2.4} & 11 & 63 & 17 & 11 & 63 & 17 & 10 & 64 & 18 & 10 & 64 & 18 & 10 & 65 & 18 \\
\hline & & 39.22218 & & & 39.674866 & & & 40.125458 & & & 40.559503 & & & 40.991876 & \\
\hline \multirow{2}{*}{2.6} & 11 & 63 & 14 & 11 & 63 & 14 & 11 & 64 & 14 & 11 & 64 & 14 & 11 & 65 & 14 \\
\hline & & 39.601437 & & & 40.053153 & & & 40.503633 & & & 40.951362 & & & 41.397236 & \\
\hline \multirow{2}{*}{2.8} & 11 & 62 & 11 & 11 & 63 & 11 & 11 & 63 & 11 & 11 & 64 & 11 & 11 & 65 & 11 \\
\hline & & 39.929298 & & & 40.380592 & & & 40.831047 & & & 41.277607 & & & 41.723459 & \\
\hline \multirow{2}{*}{3.0} & 11 & 62 & 7 & 11 & 62 & 7 & 11 & 63 & 7 & 11 & 63 & 7 & 11 & 64 & 7 \\
\hline & & 40.189174 & & & 40.641335 & & & 41.092056 & & & 41.540189 & & & 41.986075 & \\
\hline
\end{tabular}

TABLE 16: Effect of $c_{s}$ and $c_{\text {pr }}$ on the optimal cost rate $\left(c_{h}=0.3, c_{\mathrm{sh}}=5, c_{p}=3\right)$.

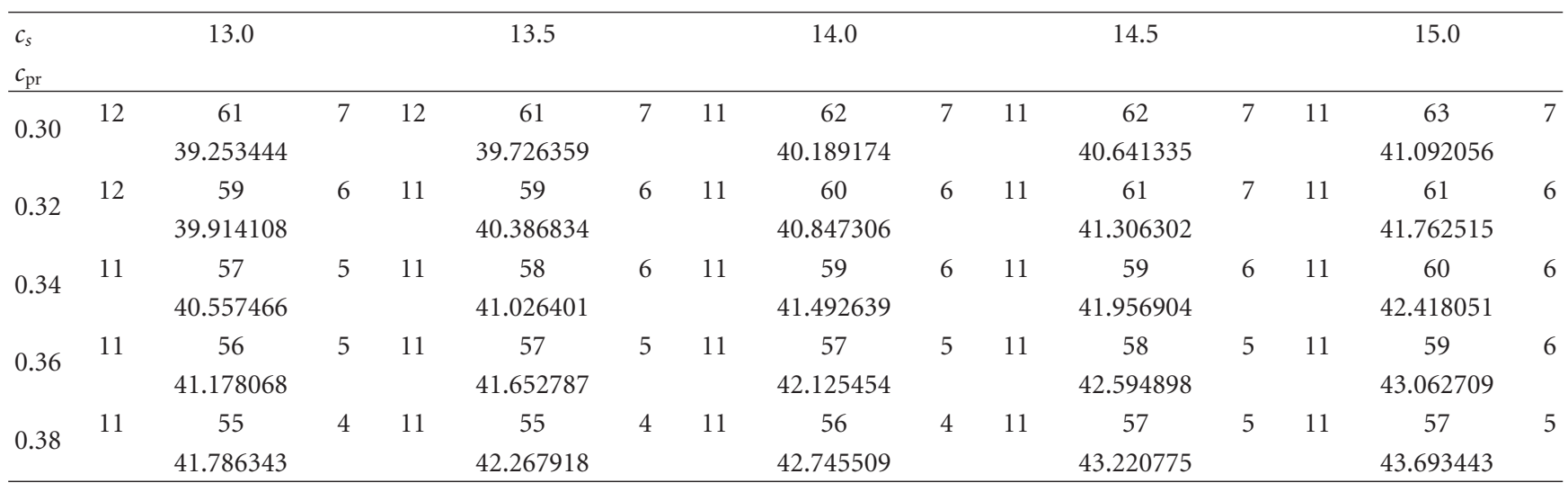

distributed with parameter $\alpha_{n}(>0)$ where $n$ is the number of customers in the pool.

Notation.

e: a column vector of appropriate dimension containing all ones.

0: a zero matrix of appropriate dimension.

$\mathbf{0}_{(i \times j)}$ : a zero matrix with $i$ rows and $j$ columns.

\section{Analysis}

Let $X(t)$ and $L(t)$, respectively, denote the number of demands in the pool and the on-hand inventory level at time $t$. We define a binary variable $Y(t)$ (which takes 0 and 1) to denote the status of the server, respectively, "on vacation" and "on service." Similarly, the binary variable $Z(t)$ will indicate the status of the reorder to replenish the stock: $Z(t)=$ 0 or 1 , respectively, if "no order is pending" or "an order is pending."

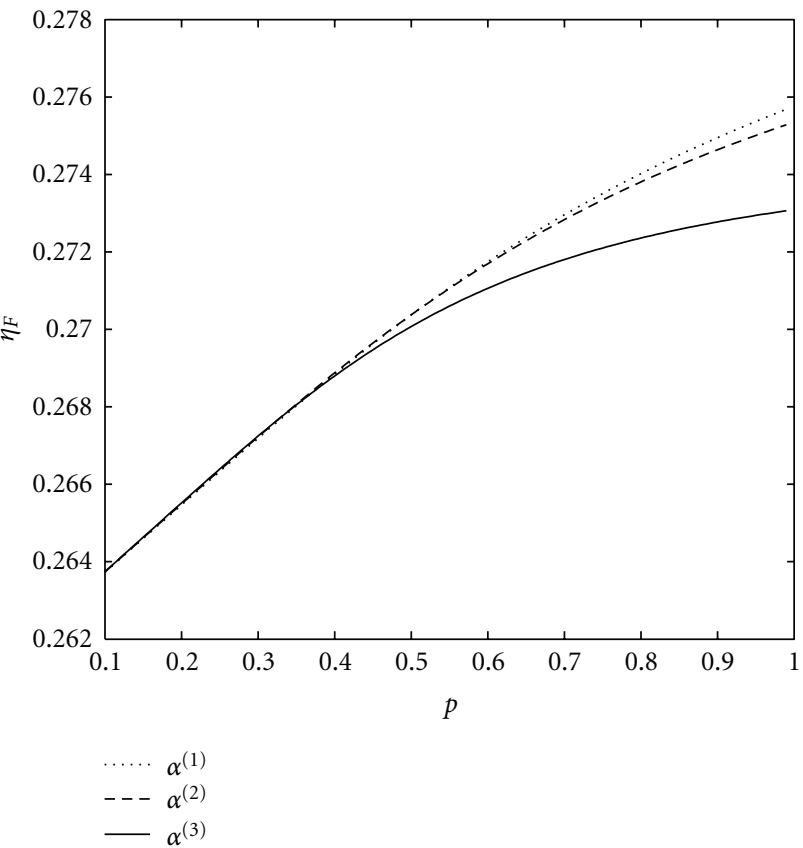

FIgURE 12: Fraction of time server is on vacation- $p$ versus $\alpha^{(n)}(S=90, s=15, N=25, \lambda=8, \beta=1.4, \gamma=0.7, \mu=0.8$. $)$ 
TABLe 17: Sensitivity of optimal values to variation in $c_{s}$ and $c_{\mathrm{sh}}\left(c_{h}=0.3, c_{p}=3, c_{\mathrm{pr}}=0.3\right)$.

\begin{tabular}{|c|c|c|c|c|c|c|c|c|c|c|c|c|c|c|c|}
\hline$c_{s}$ & & 11 & & & 12 & & & 13 & & & 14 & & & 15 & \\
\hline$c_{\mathrm{sh}}$ & & & & & & & & & & & & & & & \\
\hline 500 & 12 & 58 & 6 & 12 & 59 & 6 & 12 & 61 & 7 & 11 & 62 & 7 & 11 & 63 & 7 \\
\hline & & 37.330038 & & & 38.297398 & & & 39.253444 & & & 40.189174 & & & 41.092056 & \\
\hline 525 & 13 & 60 & 9 & 12 & 61 & 9 & 12 & 62 & 10 & 12 & 63 & 10 & 11 & 64 & 10 \\
\hline & & 37.656022 & & & 38.617201 & & & 39.561709 & & & 40.495801 & & & 41.406634 & \\
\hline 550 & 13 & 61 & 11 & 12 & 62 & 12 & 12 & 63 & 12 & 12 & 64 & 12 & 12 & 66 & 13 \\
\hline 0.00 & & 37.918543 & & & 38.88736 & & & 39.824379 & & & 40.751743 & & & 41.668858 & \\
\hline 575 & 13 & 62 & 14 & 13 & 63 & 14 & 12 & 64 & 14 & 12 & 65 & 15 & 12 & 66 & 15 \\
\hline נו. & & 38.149135 & & & 39.121004 & & & 40.056997 & & & 40.978042 & & & 41.889389 & \\
\hline 600 & 13 & 63 & 16 & 13 & 64 & 16 & 12 & 65 & 16 & 12 & 66 & 17 & 12 & 67 & 17 \\
\hline & & 38.357356 & & & 39.322828 & & & 40.267002 & & & 41.182026 & & & 42.08824 & \\
\hline
\end{tabular}

TABLE 18: Variation in optimal values for different values of $c_{p}$ and $c_{\mathrm{sh}}\left(c_{s}=15, c_{h}=0.3, c_{\mathrm{pr}}=0.3\right)$

\begin{tabular}{|c|c|c|c|c|c|c|c|c|c|c|c|c|c|c|c|}
\hline \multirow{2}{*}{$\begin{array}{l}c_{\mathrm{sh}} \\
c_{p}\end{array}$} & \multicolumn{3}{|c|}{4.8} & \multicolumn{3}{|c|}{5.0} & \multicolumn{3}{|c|}{5.2} & \multicolumn{3}{|c|}{5.4} & \multicolumn{3}{|c|}{5.6} \\
\hline & & & & & & & & & & & & & & & \\
\hline \multirow{2}{*}{2.5} & 10 & 63 & 14 & 11 & 64 & 16 & 11 & 64 & 18 & 11 & 65 & 20 & 11 & 66 & 22 \\
\hline & & 40.130057 & & & 40.320642 & & & 40.495101 & & & 40.655041 & & & 40.804415 & \\
\hline \multirow{2}{*}{2.6} & 11 & 63 & 12 & 11 & 64 & 14 & 11 & 64 & 16 & 11 & 65 & 18 & 11 & 66 & 20 \\
\hline & & 40.299295 & & & 40.503633 & & & 40.689262 & & & 40.859223 & & & 41.017575 & \\
\hline \multirow{2}{*}{2.7} & 11 & 63 & 10 & 11 & 64 & 13 & 11 & 64 & 14 & 11 & 65 & 16 & 11 & 66 & 18 \\
\hline & & 40.453574 & & & 40.674434 & & & 40.873015 & & & 41.053664 & & & 41.221074 & \\
\hline \multirow{2}{*}{2.8} & 11 & 62 & 8 & 11 & 63 & 11 & 11 & 64 & 13 & 11 & 65 & 15 & 11 & 66 & 17 \\
\hline & & 40.588856 & & & 40.831047 & & & 41.044544 & & & 41.237658 & & & 41.415324 & \\
\hline \multirow{2}{*}{2.9} & 11 & 62 & 6 & 11 & 63 & 9 & 11 & 64 & 11 & 11 & 65 & 13 & 12 & 66 & 15 \\
\hline & & 40.701943 & & & 40.97071 & & & 41.203531 & & & 41.410894 & & & 41.598786 & \\
\hline
\end{tabular}

The values taken by these random variables are listed in the following.
We consider the stochastic process $\{X(t), Y(t), Z(t), L(t), t \geq$ $0\}$. Since the components carry sufficient information on the sample path of the process and from the assumptions made on the input and output processes, this process can easily be seen to be a Markov process.

The state space of the stochastic process $\{X(t), Y(t)$, $Z(t), L(t), t \geq 0\}$ is the collection of all quadruples $\left\langle i_{1}\right\rangle=$ $\left(i_{1}, i_{2}, i_{3}, i_{4}\right)$ where each entry is selected from each column as we move from left to right; we may cross vertical lines but not horizontal ones. These quadruples can be ordered in the lexicographic order in each box separated by the horizontal lines.

We shall write the transition rate matrix in a blockpartitioned form where $i_{1}$ th row (block) corresponds to the collection of quadruples $\left\langle i_{1}\right\rangle$ (those with leftmost entry $i_{1}$ ), $i_{1}=0,1,2, \ldots, N$. It may be recalled that the pool size $\left(i_{1}\right)$ may increase or decrease by one unit (except the end states) when a demand joins or leaves the pool. Hence, the transition rate matrix is a tridiagonal one.

We also note that when the number of demands in the pool is $i(i=0,1,2, \ldots, N-1)$, a new demand joining the pool will shift the current set of quadruple $\langle i\rangle$ to $\langle i+1\rangle$ and the transition rate does not depend upon $i$. Therefore, all the entries in the upper diagonal of the rate matrix are equal to a constant block matrix, which we shall denote by $B_{0}$. The lower diagonal entries correspond to the transition from 
TABLE 19: Effect of $c_{\mathrm{sh}}$ and $c_{\mathrm{pr}}$ on the optimal cost rate $\left(c_{s}=15, c_{h}=0.3, c_{p}=3\right)$.

\begin{tabular}{|c|c|c|c|c|c|c|c|c|c|c|c|c|c|c|c|}
\hline \multirow{2}{*}{$\begin{array}{l}c_{\mathrm{sh}} \\
c_{\mathrm{pr}} \\
\end{array}$} & \multicolumn{3}{|c|}{5.0} & \multicolumn{3}{|c|}{5.2} & \multicolumn{3}{|c|}{5.4} & \multicolumn{3}{|c|}{5.6} & \multicolumn{3}{|c|}{5.8} \\
\hline & & & & & & & & & & & & & & & \\
\hline \multirow{2}{*}{0.20} & 12 & 72 & 10 & 12 & 73 & 12 & 12 & 73 & 14 & 12 & 74 & 16 & 12 & 75 & 18 \\
\hline & & 37.479871 & & & 37.668234 & & & 37.836697 & & & 37.989434 & & & 38.130738 & \\
\hline \multirow{2}{*}{0.22} & 12 & 70 & 9 & 12 & 71 & 12 & 12 & 72 & 14 & 12 & 72 & 15 & 12 & 73 & 17 \\
\hline & & 38.242077 & & & 38.439857 & & & 38.615717 & & & 38.774912 & & & 38.92153 & \\
\hline \multirow{2}{*}{0.24} & 12 & 68 & 9 & 12 & 69 & 11 & 12 & 70 & 13 & 12 & 71 & 15 & 12 & 71 & 17 \\
\hline & & 38.987028 & & & 39.193609 & & & 39.377128 & & & 39.543759 & & & 39.696724 & \\
\hline \multirow{2}{*}{0.26} & 11 & 66 & 8 & 12 & 67 & 10 & 12 & 68 & 13 & 12 & 69 & 14 & 12 & 70 & 16 \\
\hline & & 39.709844 & & & 39.932901 & & & 40.12401 & & & 40.296785 & & & 40.455714 & \\
\hline \multirow{2}{*}{0.28} & 11 & 64 & 8 & 11 & 65 & 10 & 12 & 67 & 12 & 12 & 67 & 14 & 12 & 68 & 16 \\
\hline & & 40.40882 & & & 40.653872 & & & 40.855615 & & & 41.03516 & & & 41.199655 & \\
\hline
\end{tabular}

TABLE 20: Variation in Optimal values for different values of $c_{p}$ and $c_{\mathrm{pr}}\left(c_{s}=15, c_{h}=0.3, c_{\mathrm{sh}}=5\right)$.

\begin{tabular}{|c|c|c|c|c|c|c|c|c|c|c|c|c|c|c|c|}
\hline \multirow{2}{*}{$\begin{array}{l}c_{p} \\
c_{\mathrm{pr}}\end{array}$} & \multicolumn{3}{|c|}{2.6} & \multicolumn{3}{|c|}{2.7} & \multicolumn{3}{|c|}{2.8} & \multicolumn{3}{|c|}{2.9} & \multicolumn{3}{|c|}{3.0} \\
\hline & & & & & & & & & & & & & & & \\
\hline \multirow{2}{*}{0.32} & 11 & 62 & 14 & 11 & 62 & 12 & 11 & 62 & 10 & 11 & 62 & 9 & 11 & 61 & 6 \\
\hline & & 41.181731 & & & 41.350929 & & & 41.506426 & & & 41.644627 & & & 41.762515 & \\
\hline \multirow{2}{*}{0.34} & 11 & 61 & 13 & 11 & 61 & 12 & 11 & 61 & 10 & 11 & 60 & 8 & 11 & 60 & 6 \\
\hline & & 41.846597 & & & 42.015719 & & & 42.169423 & & & 42.30555 & & & 42.418051 & \\
\hline \multirow{2}{*}{0.36} & 10 & 60 & 13 & 11 & 60 & 11 & 11 & 60 & 10 & 11 & 59 & 8 & 11 & 59 & 6 \\
\hline & & 42.4908 & & & 42.66911 & & & 42.821582 & & & 42.953905 & & & 43.062709 & \\
\hline \multirow{2}{*}{0.38} & 10 & 59 & 13 & 10 & 58 & 11 & 11 & 58 & 9 & 11 & 58 & 7 & 11 & 57 & 5 \\
\hline & & 43.120144 & & & 43.301145 & & & 43.460842 & & & 43.590387 & & & 43.693443 & \\
\hline \multirow{2}{*}{0.40} & 10 & 57 & 12 & 10 & 57 & 10 & 10 & 57 & 9 & 11 & 57 & 7 & 11 & 56 & 4 \\
\hline & & 43.737882 & & & 43.918076 & & & 44.077826 & & & 44.215885 & & & 44.3119 & \\
\hline
\end{tabular}

states of $\langle i\rangle, i=1,2, \ldots, N$, to states $\langle i-1\rangle$. This is due to the selection of a demand from the pool, and the rate of selection of any one of the demands is $\alpha_{i}$. Hence, these transition rates depend on row indices. Therefore, the entries of the lower diagonal depend on the row index. We shall denote them by $C_{i}$. In the case of diagonal entries, again, the transition rate may depend upon the pool size (row index), if it involves selection of a demand from the pool. Hence, the entries here also depend upon the respective row index. We shall denote them by $A_{i}$.

Hence, the infinitesimal generator matrix can be written as

$$
\begin{aligned}
& \begin{array}{lllllll}
0 & 1 & 2 & 3 & \cdots & N-2 & N-1 \quad N
\end{array} \\
& \left.\begin{array}{c|cccccccc}
0 & A_{0} & B_{0} & 0 & 0 & \cdots & \cdots & 0 & 0 \\
1 & C_{1} & A_{1} & B_{0} & 0 & \cdots & \cdots & 0 & 0 \\
2 & 0 & C_{2} & A_{2} & B_{0} & \cdots & \cdots & 0 & 0 \\
\vdots & \vdots & \vdots & \vdots & \vdots & \vdots & \vdots & \vdots & \vdots \\
N-2 & \cdots & \cdots & \ldots & \ldots & \cdots & A_{N-2} & B_{0} & \cdots \\
N-1 & \ldots & \ldots & \ldots & \ldots & \cdots & C_{N-1} & A_{N-1} & B_{0} \\
N & \ldots & \ldots & \ldots & \ldots & \cdots & 0 & C_{N} & A_{N}
\end{array}\right) .
\end{aligned}
$$

We shall determine the submatrix $B_{0}$, the elements of which correspond to the transitions from $\langle i\rangle$ to $\langle i+1\rangle, i=$ $0,1,2, \ldots, N-1$. This corresponds to the case that one demand has joined the pool. In a Markov process, at most one event can occur in a small interval. Hence, for the transitions from $\left(i_{1}, i_{2}, i_{3}, i_{4}\right)$ to $\left(i_{1}+1, i_{2}^{\prime}, i_{3}^{\prime}, i_{4}^{\prime}\right)$, we have 0 when $i_{2}^{\prime} \neq i_{2}, i_{3}^{\prime} \neq i_{3}, i_{4}^{\prime} \neq i_{4}$ and $p \lambda$ when they are equal and $i_{2}=0$. The matrix $B_{0}$ is given by

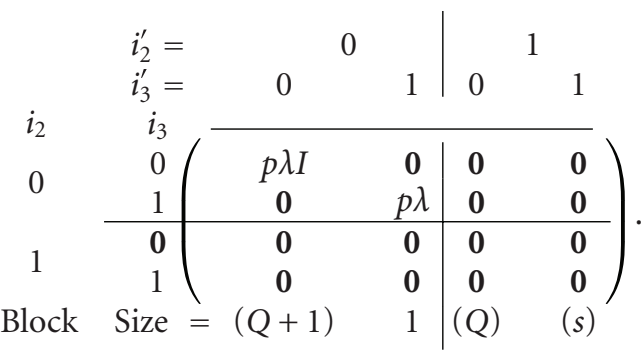

Next, we consider the submatrix $C_{i}$, the elements of which give the transitions from $\langle i\rangle$ to $\langle i-1\rangle$. This corresponds to the event that a demand from the pool is selected, and this indicates that the server is "on service" and that "no order is pending." Therefore, possible value is $i_{2}^{\prime}=i_{2}=1$. As a result of a selection from the pool, an item from the inventory is used to meet this demand. Hence, $L(t)$ moves from $i_{4}$ to $i_{4}-1$, if $i_{4}=s+2, s+3, \ldots, S$ (with $i_{3}^{\prime}=0$ ) 
and from $s+1$ to $s$ (with $i_{3}^{\prime}=1$ ). Hence, the submatrix $C_{i}, i=1,2, \ldots, N$, is given by

$\begin{array}{cccc|ccc} & \begin{array}{c}i_{2}^{\prime}= \\ i_{3}^{\prime}=\end{array} & & 0 & & & 1 \\ i_{2} & i_{3} & & & & \\ & & & & & \\ 0 & 0 & \mathbf{0} & \mathbf{0} & \mathbf{0} & \mathbf{0} \\ & 1 & \mathbf{0} & \mathbf{0} & \mathbf{0} & \mathbf{0} \\$\cline { 2 - 5 } 1 & 0 & $\left.\mathbf{0} & \mathbf{0} & C_{0}^{(i)} & C_{0}^{(i)} \\ \text { Block } & 1 & \mathbf{0} & \mathbf{0} & \mathbf{0} & \mathbf{0}\end{array}\right)$,

where

$$
\begin{aligned}
C_{0}^{(i)} & =\left(\begin{array}{ccccc}
0 & 0 & \cdots & 0 & 0 \\
\alpha_{i} & 0 & \cdots & 0 & 0 \\
0 & \alpha_{i} & \cdots & 0 & 0 \\
\vdots & \vdots & \vdots & \vdots & \vdots \\
0 & 0 & \cdots & \alpha_{i} & 0
\end{array}\right), \\
C_{1}^{(i)} & =\left(\begin{array}{ccccc}
0 & 0 & \cdots & 0 & \alpha_{i} \\
0 & 0 & \cdots & 0 & 0 \\
0 & 0 & \cdots & 0 & 0 \\
\vdots & \vdots & \vdots & \vdots & \vdots \\
0 & 0 & \cdots & 0 & 0
\end{array}\right) .
\end{aligned}
$$

Let us consider the submatrix $A_{i}, i=1,2, \ldots, N$. In this case, there is no addition to nor selection from the pool. Firstly, we note the transitions

(i) from $\left(i_{1}, 0,1, i_{4}\right)$ to $\left(i_{1}, 1,0, i_{4}\right)$ or $\left(i_{1}, 1,1, i_{4}\right)$,

(ii) from $\left(i_{1}, 1,0, i_{4}\right)$ to $\left(i_{1}, 0,0, i_{4}\right)$ or $\left(i_{1}, 0,1, i_{4}\right)$,

(iii) from $\left(i_{1}, 1,1, i_{4}\right)$ to $\left(i_{1}, 0,0, i_{4}\right)$,

as these involve more than one occurrence.

Secondly, we observe that, only in the case of diagonal entries $a\left(\left(i_{1}, 1,0, i_{4}\right),\left(i_{1}, 1,0, i_{4}\right)\right)$ (i.e., the intensity of passage), the rate $\alpha_{i}$ (the rate of selection from the pool) will appear.

Hence, the block-partitioned submatrix $A_{i}$ will have the following structure:

$$
\begin{array}{cccc|ccc} 
& \begin{array}{c}
i_{2}^{\prime}= \\
i_{3}^{\prime}=
\end{array} & & 0 & & \multicolumn{2}{|c}{1} \\
i_{2} & i_{3} & & 1 & 0 & 1 \\
\cline { 3 - 6 } 0 & 0 & A_{00} & a_{01} & F_{00} & F_{01} \\
& 1 & a_{10} & a_{11} & \mathbf{0} & \mathbf{0} \\
\cline { 2 - 6 } 1 & 0 & \mathbf{0} & \mathbf{0} & H_{00}^{(i)} & H_{01} \\
\text { Block } & \text { Size }=(Q+1) & 1 & (Q) & (s)
\end{array}
$$

The following cases are pertaining to the event that the server is on vacation before and after the transition. (i) In the case of column vector $a_{01}$, the reorder status changes from "no order is pending" to "an order is pending." Thus, $i_{4}=0$ and, after transition, $i_{4}^{\prime}=$ 0 . No transition is possible from other states $i_{4}=$ $1,2, \ldots, Q$. Thus, we obtain

$$
a_{01}=(\beta, 0, \ldots, 0)^{\prime}
$$

(ii) The row vector $a_{10}$ considers the change of reorder status (from "order is pending" to "no order is pending"). Thus, a supply of $Q$ items is received. This changes $i_{2}=0, i_{3}=0, i_{4}=0$ to $i_{2}^{\prime}=0, i_{3}^{\prime}=1, i_{4}^{\prime}=$ Q. Hence,

$$
a_{10}=(0,0, \ldots, 0, \mu)
$$

(iii) The constant $a_{11}$ is a diagonal entry, and, considering all transitions from $\left(i_{1}, 0,1,0\right)$ to other states, we get

$a_{11}= \begin{cases}-(p \lambda+\mu) & \text { if } i_{1}=1,2, \ldots, N-1, \\ -\mu & \text { if } i_{1}=N\end{cases}$

or simply $a_{11}=-\left(p \lambda \bar{\delta}_{i_{1} N}+\mu\right)$, where $\bar{\delta}_{i_{1} N}=1-\delta_{i_{1} N}$.

(iv) The nondiagonal entry of $A_{00}$, namely, $a\left(\left(i_{1}, 0,0, i_{4}\right),\left(i_{1}, 0,0, i_{4}^{\prime}\right)\right)$ is equal to $i_{4} \gamma$ for $i_{4}^{\prime}=i_{4}-1$ and zero for other nondiagonal elements. For the diagonal entry (considering the transitions to other states), we get

$\left[A_{00}\right]_{i_{4}, i_{4}}= \begin{cases}-\left(p \lambda+\beta+i_{4} \gamma\right) & \text { if } i_{4}=1,2, \ldots, Q, i_{1} \neq N, \\ -\left(\beta+i_{4} \gamma\right) & \text { if } i_{4}=1,2, \ldots, Q, i_{1}=N,\end{cases}$

\begin{tabular}{|c|c|c|c|c|c|}
\hline & $i_{2}^{\prime}=$ & \multicolumn{2}{|c|}{0} & \multicolumn{2}{|c|}{1} \\
\hline & $i_{3}^{\prime}=$ & 0 & 1 & 0 & 1 \\
\hline 0 & $\begin{array}{l}0 \\
1\end{array}$ & $\begin{array}{c}D_{00}^{(i)} \\
\mu u_{Q+1}^{\prime}\end{array}$ & $\begin{array}{c}\beta u_{1} \\
-\left(\mu+p \lambda \bar{\delta}_{i N}\right)\end{array}$ & $\begin{array}{c}F_{00} \\
\mathbf{0}\end{array}$ & $\begin{array}{c}F_{01} \\
\mathbf{0}\end{array}$ \\
\hline 1 & $\begin{array}{l}0 \\
1\end{array} \mid$ & $\begin{array}{l}\mathbf{0} \\
\mathbf{0}\end{array}$ & $\begin{array}{c}\mathbf{0} \\
(\lambda+\gamma) u_{1}\end{array}$ & $\begin{array}{l}H_{00}^{(i)} \\
H_{10}\end{array}$ & $\begin{array}{l}H_{01} \\
H_{11}\end{array}$ \\
\hline Block & Size $=$ & $(Q+1)$ & 1 & $(Q)$ & $(s)$ \\
\hline
\end{tabular}

or, simply, $-\left(p \lambda \bar{\delta}_{i_{1} N}+\beta+i_{4} \gamma\right)$ if $i_{4}=1,2, \ldots, Q$, for all $i_{1}$.

Using arguments similar to the above, we can express the matrix $A_{i}, i=1,2, \ldots, N$ as follows:

where

(i) $u_{k}^{\prime}=(0,0, \ldots, 1, \ldots, 0,0)$ a vector having 1 at $k$ th place and 0 at other places, 
(ii)

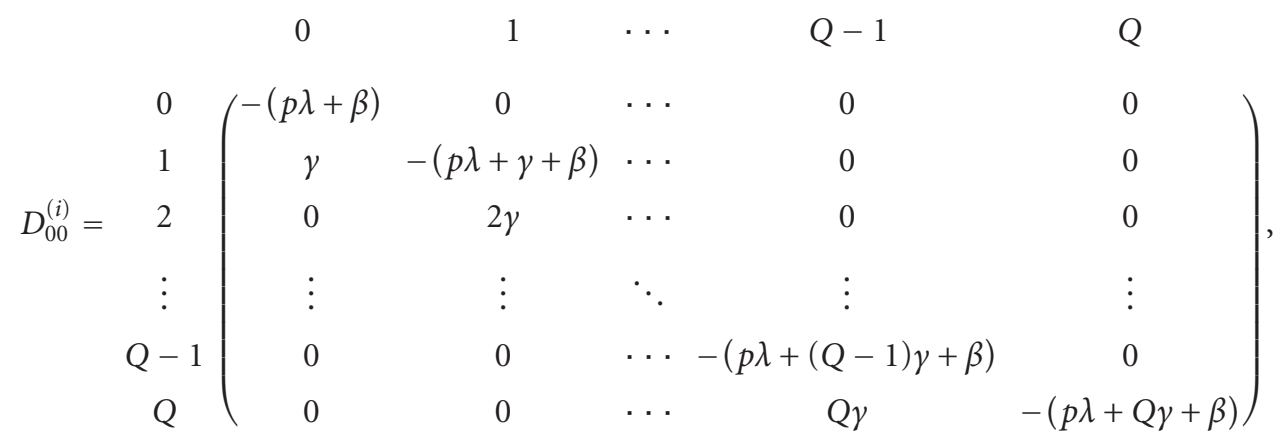

(iii)

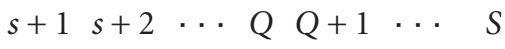

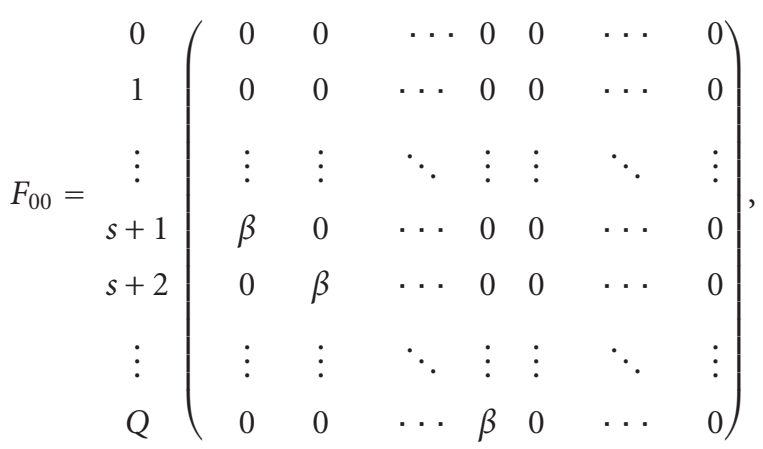

(iv)

$$
\begin{aligned}
& \begin{array}{lllll}
1 & 2 & \cdots & s-1 & s
\end{array}
\end{aligned}
$$

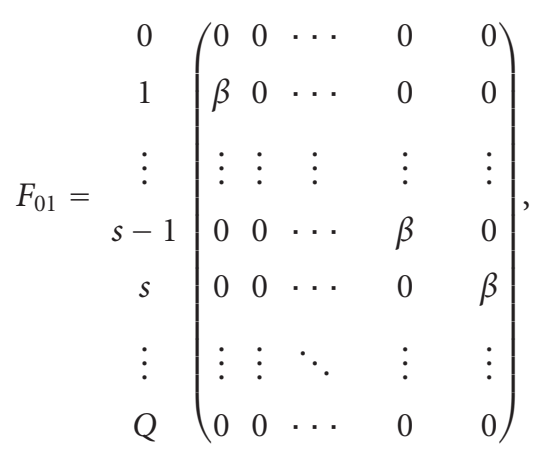

(v)

$$
\begin{aligned}
& \begin{array}{lllll}
s+1 & s+2 & \cdots & S-1 & S
\end{array}
\end{aligned}
$$

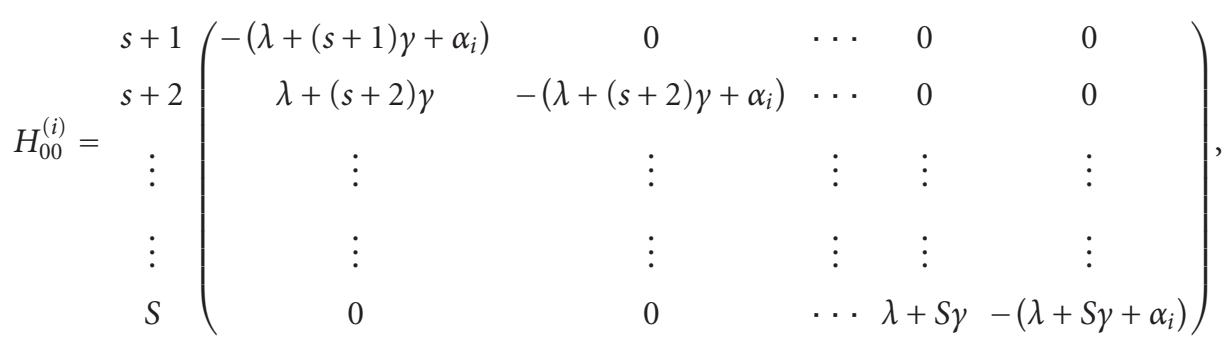


(vi)

$$
\begin{gathered}
1 \\
H_{01}=s+1 \\
s+2 \\
\vdots \\
S
\end{gathered}\left(\begin{array}{ccccc}
0 & 0 & \cdots & 0 & \lambda+(s+1) \gamma \\
0 & 0 & \cdots & 0 & 0 \\
\vdots & \vdots & \cdots & \vdots & \vdots \\
0 & 0 & \cdots & 0 & 0
\end{array}\right),
$$

(vii)

$$
H_{10}={ }_{s}{ }^{1}\left(\begin{array}{cccccc}
0 & \cdots & 0 & \mu & \cdots & 0 \\
\vdots & \ddots & \cdots & \vdots & \ddots & \vdots \\
0 & \cdots & 0 & 0 & \cdots & \mu
\end{array}\right) \text {, }
$$

(viii)

$$
H_{11}=\begin{array}{ccccc}
1 & 2 & \cdots & s-1 & s \\
& 1 \\
& \vdots
\end{array}\left(\begin{array}{ccccc}
-(\lambda+\gamma+\mu) & 0 & \cdots & 0 & 0 \\
\lambda+2 \gamma & -(\lambda+2 \gamma+\mu) & \cdots & 0 & 0 \\
\vdots & \vdots & \vdots & \vdots & \vdots \\
0 & 0 & \cdots & \lambda+s \gamma & -(\lambda+s \gamma+\mu)
\end{array}\right) .
$$

\section{Steady-State Analysis}

It can be seen from the structure of the infinitesimal generator $A$ that the time-homogeneous Markov process $\{(X(t), Y(t), Z(t), L(t)) ; t \geq 0\}$ on the finite state space $E$ is irreducible. Hence, the limiting distribution

$$
\begin{gathered}
\phi^{\left(j_{1}, j_{2}, j_{3}, j_{4}\right)}=\lim _{t \rightarrow \infty} \operatorname{Pr}\left[X(t)=j_{1}, Y(t)=j_{2},\right. \\
Z(t)=j_{3}, L(t)=j_{4} \mid X(0), Y(0), Z(0), \\
L(0)]
\end{gathered}
$$

exists. Let

$$
\begin{aligned}
\phi^{\left(i_{1}\right)}=( & \phi^{\left(i_{1}, 0,0,0\right)}, \phi^{\left(i_{1}, 0,0,1\right)}, \ldots, \phi^{\left(i_{1}, 0,0, Q\right)}, \\
& \phi^{\left(i_{1}, 0,1,0\right)}, \phi^{\left(i_{1}, 1,0, s+1\right)}, \phi^{\left(i_{1}, 1,0, s+2\right)}, \ldots, \phi^{\left(i_{1}, 1,0, S\right)}, \\
& \left.\phi^{\left(i_{1}, 1,0, s+1\right)}, \phi^{\left(i_{1}, 1,0, s+2\right)}, \ldots, \phi^{\left(i_{1}, 1,0, S\right)}\right), \\
& \phi^{\left(i_{1}, 1,1,1\right)}, \phi^{\left(i_{1}, 1,1,2\right)}, \ldots, \phi^{\left(i_{1}, 1,1, s\right)}, \\
\Phi= & \left(\phi^{(0)}, \phi^{(1)}, \ldots, \phi^{(N)}\right) .
\end{aligned}
$$

Then, the vector of limiting probabilities $\Phi$ satisfies

$$
\Phi A=\mathbf{0}, \quad \Phi \mathbf{e}=1
$$

From the structure of $A$, it is seen that the Markov process under study falls into the class of birth and death process in a Markovian environment as discussed by Gaver et al. [25]. Hence, using the same arguments, we can calculate the limiting probability vectors. For the sake of completeness, we provide the algorithm here.
Algorithm. (1) Determine recursively the matrices

$$
\begin{aligned}
& F_{0}=A_{0} \\
& F_{i_{1}}=A_{i_{1}}+C_{i_{1}}\left(-F_{i_{1}-1}^{-1}\right) B_{0}, \quad i_{1}=1,2, \ldots, N .
\end{aligned}
$$

(2) Compute recursively the vectors $\phi_{\left(i_{1}\right)}$ using

$$
\phi^{\left(i_{1}\right)}=\phi^{\left(i_{1}+1\right)} C_{i_{1}+1}\left(-F_{i_{1}}^{-1}\right), \quad i_{1}=N-1, N-2, \ldots, 0 .
$$

(3) Solve the system of equations

$$
\begin{aligned}
\phi^{(N)} F_{N} & =\mathbf{0} \\
\sum_{i_{1}=0}^{N} \phi^{\left(i_{1}\right)} \mathbf{e} & =1 .
\end{aligned}
$$

From the system of (24), vector $\phi^{(N)}$ could be determined uniquely, up to a multiplicative constant. This constant is obtained by using (23) and (25).

\section{System Performance Measures}

In this section, we give some system performance measures, which are useful in the qualitative interpretation of the model under study.

5.1. Expected Inventory Level. Let $\eta_{I}$ denote the mean inventory level in the steady-state. Since $\phi^{(i, k, p, m)}$ denotes the steady state probability when the number of demands in the 
pool is $i$, the server status is $k$, the status of reorder is $p$, and the inventory level is $m$, the mean inventory level is given by

$$
\begin{gathered}
\eta_{I}=\sum_{i=0}^{N}\left(\sum_{m=1}^{Q} m \phi^{(i, 0,0, m)}+\sum_{m=s+1}^{S} m \phi^{(i, 1,0, m)}\right. \\
\left.+\sum_{m=1}^{s} m \phi^{(i, 1,1, m)}\right) .
\end{gathered}
$$

5.2. Expected Reorder Rate. Let $\eta_{R}$ denote the expected reorder rate in the steady state. We note that a reorder is triggered

(i) when the inventory level drops from $s+1$ to $s$ if the server is in service or

(ii) when the server returns from the vacation, he/she finds the inventory level less than or equal to $s$ and there is no order pending.

This leads to

$$
\begin{aligned}
\eta_{R}= & \sum_{i=0}^{N} \phi^{(i, 1,0, s+1)}\left(\lambda+(s+1) \gamma+\bar{\delta}_{i 0} \alpha_{i}\right) \\
& +\sum_{i=0}^{N} \sum_{m=0}^{s} \beta \phi^{(i, 0,0, m)}
\end{aligned}
$$

where $\delta_{i j}$ is the Kronecker delta function.

5.3. Expected Perishable Rate. Let $\eta_{\mathrm{PR}}$ denote the expected reorder Perishable in the steady state. This is given by

$$
\begin{gathered}
\eta_{\mathrm{PR}}=\sum_{i=0}^{N}\left(\sum_{m=1}^{Q} m \gamma \phi^{(i, 0,0, m)}+\sum_{m=s+1}^{S} m \gamma \phi^{(i, 1,0, m)}\right. \\
\left.+\sum_{m=1}^{s} m \gamma \phi^{(i, 1,1, m)}\right) .
\end{gathered}
$$

5.4. Mean Shortage Rate. Let $\eta_{\mathrm{SR}}$ denote the mean shortage rate in the steady state. It may be noted that a shortage occurs only during the server vacation. The customers who arrive during server vacation prefer to leave the system either by their own choice or the pool has reached its maximum capacity:

$$
\begin{aligned}
\eta_{\mathrm{SR}}= & \sum_{i=0}^{N-1} q \lambda\left[\sum_{m=0}^{Q} \phi^{(i, 0,0, m)}+\phi^{(i, 0,1,0)}\right] \\
& +\lambda\left[\sum_{m=0}^{Q} \phi^{(N, 0,0, m)}+\phi^{(N, 0,1,0)}\right] .
\end{aligned}
$$

5.5. Expected Number of Customers in the Pool. Let $\eta_{P}$ denote the expected number of customers in the pool in the steady state. Since $\phi^{(i)}$ is the steady state probability vector for $i$ customers in the pool with each component specifying the probability for the particular combination of the status of the server, status of the reorder, and the inventory level, the expected number of customers in the pool is given by

$$
\eta_{P}=\sum_{i=1}^{N} i \phi^{(i)} \mathbf{e}
$$

5.6. Fraction of Time When the Server is on Vacation. Let $\eta_{F}$ denote the fraction of time when the server is on vacation. This is given by

$$
\eta_{F}=\sum_{i=0}^{N} \sum_{m=0}^{Q} \phi^{(i, 0,0, m)}+\sum_{i=0}^{N} \phi^{(i, 0,1,0)} .
$$

\section{Cost Analysis}

The expected total cost per unit time (expected total cost rate) in the steady state for this model is defined to be

$$
\begin{aligned}
\mathrm{TC}(s, S, N)= & c_{h} \eta_{I}+c_{p} \eta_{P}+c_{\mathrm{pr}} \eta_{\mathrm{PR}} \\
& +c_{s} \eta_{R}+c_{\mathrm{sh}} \eta_{\mathrm{SR}},
\end{aligned}
$$

where

$c_{s}:$ setup cost per order,

$c_{h}$ : the inventory carrying cost per unit item per unit time,

$c_{p}$ : cost of a waiting customer in the pool per unit time,

$c_{\mathrm{pr}}$ : perishable cost per unit item per unit time,

$c_{\mathrm{sh}}$ : cost per customer lost per unit time.

Hence, we get

$$
\begin{aligned}
& \mathrm{TC}(s, S, N)=c_{h}\left\{\sum _ { i = 0 } ^ { N } \left(\sum_{m=1}^{Q} m \phi^{(i, 0,0, m)}+\sum_{m=s+1}^{S} m \phi^{(i, 1,0, m)}\right.\right. \\
& \left.\left.+\sum_{m=1}^{s} m \phi^{(i, 1,1, m)}\right)\right\}+c_{p}\left\{\sum_{i=1}^{N} i \phi^{(i)} \mathbf{e}\right\} \\
& +c_{s}\left\{\sum_{i=0}^{N} \phi^{(i, 1,0, s+1)}\left(\lambda+(s+1) \gamma+\bar{\delta}_{i 0} \alpha_{i}\right)\right. \\
& \left.+\sum_{i=0}^{N} \sum_{m=0}^{s} \beta \phi^{(i, 0,0, m)}\right\} \\
& +c_{\mathrm{pr}}\left\{\sum _ { i = 0 } ^ { N } \left(\sum_{m=0}^{Q} m \gamma \phi^{(i, 0,0, m)}+\sum_{m=s+1}^{S} m \gamma \phi^{(i, 1,0, m)}\right.\right. \\
& \left.\left.+\sum_{m=1}^{s} m \gamma \phi^{(i, 1,1, m)}\right)\right\} \\
& +c_{\mathrm{sh}}\left\{\sum_{i=0}^{N-1} q \lambda\left[\sum_{m=0}^{Q} \phi^{(i, 0,0, m)}+\phi^{(i, 0,1,0)}\right]\right. \\
& \left.+\lambda\left[\sum_{m=0}^{Q} \phi^{(N, 0,0, m)}+\phi^{(N, 0,1,0)}\right]\right\} \text {. }
\end{aligned}
$$


Due to the complex form of the limiting distribution, it is difficult to discuss the properties of the cost function $\mathrm{TC}(s, S, N)$ analytically. Hence, a detailed computational study of the expected cost function is carried out in the next section.

\section{Numerical Illustrations}

To study the behaviour of the model developed in this work, several examples were performed and a set of representative results is shown here. Although we have not shown the convexity of $\operatorname{TC}(s, S, N)$ analytically, our experience with considerable numerical examples indicates the function $\mathrm{TC}(s, S, N)$ to be convex. In some cases, it turned out to be an increasing function of $s$, and we use simple numerical search procedure to get the optimal values of TC, $s, S$ and $N$ (say $\mathrm{TC}^{*}, s^{*}, S^{*}$, and $\left.N^{*}\right)$. A typical three-dimensional plot of the expected cost function is given in Figure 1. The optimal cost value $\mathrm{TC}^{*}=37.352776$ is obtained at $\left(s^{*}, S^{*}, N^{*}\right)=$ $(11,63,11)$.

We have studied the effect of varying the system parameters and costs on the optimal values, and the results agreed with what one would expect. Some of our results are presented in Tables 1 through 20 where the upper entries in each cell give the $s^{*}, S^{*}$, and $N^{*}$, respectively, and the lower entry gives the corresponding $\mathrm{TC}^{*}$.

Example 1. In the first example, we study the impact of the demand rate $\lambda$, the lead time rate $\mu$, the perishable rate $\gamma$, the selection rate $\alpha_{i}$, and the vacation time parameter $\beta$ on the optimal values $s^{*}, S^{*}, N^{*}$, and $\mathrm{TC}^{*}$. Towards this end, we first fix $\alpha_{i}=i \alpha, i=1,2, \ldots, N$, and the cost values as $c_{h}=0.3, c_{s}=15, c_{\mathrm{pr}}=0.3, c_{\mathrm{sh}}=5$, and $c_{p}=3$. We observe the following from Table 1 to 10 .

(1) The optimal values $s^{*}, S^{*}$, and $\mathrm{TC}^{*}$ increase monotonically with increase in $\lambda$. This is to be expected since, when the arrivals occurs closer and closer, the optimal reorder level and the maximum inventory level increase to prevent more waiting time of the demands in the pool. We also note that, as $\lambda$ increases, $N^{*}$ increases except for the case in which $\gamma$ increases.

(2) As is to be expected, the optimal cost rate $\mathrm{TC}^{*}$ decreases monotonically, when $\mu, \alpha$, and $\beta$ increase and monotonically increases when $\gamma$ increases.

(3) When $\mu$ increases, $s^{*}$ and $S^{*}$ decrease and $N^{*}$ increases. This can be explained intuitively as follows. If the replenishments occur further and further apart, the inventory level becomes zero frequently. During the stock-out period, a part of arriving demands enter into the pool. To prevent the loss of demand, we have necessity to increase the pool size. For the same reason as $\beta$ increases, $s^{*}$ and $S^{*}$ decrease and $N^{*}$ increases.

(4) We note that, as $\gamma$ increases, $s^{*}$ and $N^{*}$ decrease monotonically. We also note that except for the combination of increasing $\gamma, S^{*}$ decreases when $\alpha$ increases.
(5) As $\alpha$ increases, $N^{*}$ increases and $s^{*}$ decreases. We also note that, except for the combination of increasing $\mu$, $S^{*}$ increases when $\alpha$ increases.

Example 2. In this example, we study the impact of the setup cost $c_{s}$, holding cost $c_{h}$, perishable cost $c_{\mathrm{pr}}$, shortage cost $c_{\mathrm{sh}}$, and the waiting $\operatorname{cost} c_{p}$ on the optimal values $s^{*}, S^{*}, N^{*}$, and $\mathrm{TC}^{*}$. Towards this end, we first fix the parameter values as $\lambda=14, \gamma=1.4, \beta=1.3, \alpha_{i}=i \alpha, i=1,2, \ldots, N, \alpha=$ $4, \mu=4$, and $p=0.85$. We observe the following from Table 11 to 20 :

(1) The optimal cost increases, when $c_{h}, c_{s}, c_{\mathrm{pr}}, c_{\mathrm{sh}}$, and $c_{p}$ increase. The optimal cost is more sensitive to $c_{h}$ than to $c_{s}, c_{\mathrm{pr}}, c_{\mathrm{sh}}$, and $c_{p}$.

(2) As $c_{h}$ increases, as is to be expected, the optimal values $s^{*}, S^{*}$, and $N^{*}$ decrease monotonically. This is to be expected since the holding cost increases, we resort to maintain low stock in the inventory.

(3) When $c_{\text {sh }}$ increases, as is to be expected, the optimal values $s^{*}, S^{*}$, and $N^{*}$ increase monotonically. This is to be expected since for the high shortage cost to reduce the number of customer to be lost, we have to increase the pool size, maintain high inventory, and place order in the higher level.

(4) If the waiting cost $c_{p}$ increases, the optimal values of $s^{*}$ increase and $N^{*}$ decreases monotonically. We also note that, except for the combination of increasing the holding cost $c_{h}, S^{*}$ decreases when $c_{p}$ increases.

(5) If the setup cost $c_{s}$ increases, it is a common decision that we have to maintain more stock to avoid frequent ordering. This fact is also observed in our model.

(6) We note that, when the perishable cost $c_{\mathrm{pr}}$ increases $s^{*}, S^{*}$, and $N^{*}$ decrease monotonically.

Example 3. In this example, we look to the impact of the demand rate $\lambda$, the reorder rate $\mu$, the selection rate $\alpha$, and the mean vacation time $1 / \beta$ on the fraction, $\eta_{F}$, of time the server is on vacation for the combination of various values of postponed demand rate $\alpha^{(n)}(n=1,2,3), \alpha^{(n)}$ is the increasing, constant, and decreasing functions, where $\alpha^{(1)}=$ $5+i * 0.1, \alpha^{(2)}=5$, and $\alpha^{(3)}=5+i *(-0.1), i=1, \ldots, N$. From Figures 2 to 5, we observe the following.

(i) As is to be expected as $\lambda$ increases, $\eta_{F}$ increases. This is because, if the demands rate increases, the stockout occurs frequently, and hence the server takes vacation frequently. Hence, $\eta_{F}$ increases. Similarly the same thing is observed in the case $\gamma$.

(ii) As $\mu$ increases, $\eta_{F}$ decreases. This happens because, if $\mu$ increases, the ordered items are received at higher rate. Hence, fewer stock-out periods occur. Hence the server returns to the system more often. Hence, the intervacation time increases. Therefore $\eta_{F}$ decreases. Similarly, the same thing is observed in the case $\beta$.

(iii) In these cases, we observe $\alpha^{(1)}>\alpha^{(2)}>\alpha^{(3)}$. 
Example 4. In this example, we now study the impact of $p$ on the performance measures, expected Inventory level $\eta_{I}$, expected reorder rate $\eta_{R}$, expected perishable rate $\eta_{\mathrm{PR}}$, mean shortage rate $\eta_{\mathrm{SR}}$, expected number of customers in the pool $\eta_{P}$, fraction of time the server is on vacation $\eta_{F}$ and the total expected cost $\mathrm{TC}(s, S, N)$, for the combination of various values of postponed demand rate $\alpha^{(n)}(n=1,2,3)$, $\alpha^{(n)}$ is the increasing, constant, and decreasing functions, where $\alpha^{(1)}=5+i * 0.1, \alpha^{(2)}=5$ and $\alpha^{(3)}=5+i *(-0.1)$, $i=1, \ldots, N$. From Figure 6 to 12, we observe the following.

(i) As $\mathrm{TC}(s, S, N), \eta_{\mathrm{SR}}$ and $\eta_{P}$ are monotonically increasing, when $p$ increases. In these cases, we observe $\alpha^{(1)}<\alpha^{(2)}<\alpha^{(3)}$.

(ii) As $\eta_{R}$ and $\eta_{F}$ are monotonically increasing, when $p$ increases. In these cases, we observe $\alpha^{(1)}>\alpha^{(2)}>\alpha^{(3)}$.

(iii) As $\eta_{I}$ and $\eta_{\mathrm{PR}}$ are monotonically decreasing, when $p$ increases. In these cases, we observe $\alpha^{(1)}<\alpha^{(2)}<\alpha^{(3)}$.

\section{Acknowledgments}

The authors wish to thank the anonymous referee for the comments, which significantly improved the presentation of this paper. G. Arivarignan would like to thank the University Grants Commission (UGC), India, for their financial support (F. no. 32-170/2006(SR)). B. Sivakumar would like to thank the Council of Scientific and Industrial Research (CSIR)-India for their financial support (no. 25(0813)/10/EMR-II).

\section{References}

[1] S. Nahmias, "Perishable inventory theory: a review," Operations Research, vol. 30, no. 4, pp. 680-708, 1982.

[2] F. Raafat, "Survey of literature on continuously deteriorating inventory models," Journal of the Operational Research Society, vol. 42, no. 1, pp. 27-37, 1991.

[3] N. H. Shah and Y. K. Shah, "Literature survey on inventory models for deteriorating items," Ekonomski Anali, vol. 44, pp. 221-237, 2000.

[4] S. K. Goyal and B. C. Giri, "Recent trends in modeling of deteriorating inventory," European Journal of Operational Research, vol. 134, no. 1, pp. 1-16, 2001.

[5] S. R. Chakravarthy and J. K. Daniel, "A Markovian inventory system with random shelf time and back orders," Computers and Industrial Engineering, vol. 47, no. 4, pp. 315-337, 2004.

[6] V. S. S. Yadavalli, W. Van Schoor Cde, J. J. Strashein, and S. Udayabakaran, "A single product perishing inventory model with demand interaction," ORiON, vol. 20, pp. 109-124, 2004.

[7] S. Kalpakam and S. Shanthi, "Perishable inventory system with modified $(\mathrm{S}-1, \mathrm{~S})$ policy and arbitrary processing times," Computers and Operations Research, vol. 28, no. 5, pp. 453471, 2001.

[8] S. Kalpakam and S. Shanthi, "A continuous review perishable system with renewal demands," Annals of Operations Research, vol. 143, no. 1, pp. 211-225, 2006.

[9] Z. Lian and L. Liu, "Continuous review perishable inventory systems: models and heuristics," IIE Transactions, vol. 33, no. 9, pp. 809-822, 2001.
[10] L. Liu and D. H. Shi, " $(s, S)$ model for inventory with exponential lifetimes and renewal demands," Naval Research Logistics, vol. 46, no. 1, pp. 39-56, 1999.

[11] S. Kalpakam and G. Arivarignan, "A continuous review perishable inventory model," Statistics, vol. 19, no. 3, pp. 389398, 1988.

[12] Ü. Gürler and B. Y. Özkaya, "A note on 'continuous review perishable inventory systems: models and heuristics", IIE Transactions, vol. 35, no. 3, pp. 321-323, 2003.

[13] J. K. Daniel and R. Ramanarayanan, "An inventory system with two servers and rest periods," in Cahiers du Centre d'études de recherche opérationnelle, vol. 29, pp. 95-100, Universite Libre De Bruxelles, 1987.

[14] J. K. Daniel and R. Ramanarayanan, "An $(s, S)$ inventory system with rest periods to the server," Naval Research Logistics, vol. 35, no. 1, pp. 119-123, 1988.

[15] B. T. Doshi, "Queueing systems with vacations-a survey," Queueing Systems, vol. 1, no. 1, pp. 29-66, 1986.

[16] B. T. Doshi, "Single server queues with vacations," in Stochastic Analysis of Computer and Communication Systems, H. Takagi, Ed., pp. 217-265, Elsevier, Amsterdam, The Netherlands, 1990.

[17] H. Takagi, Queueing Analysis-A Foundation of Performance Evaluation, vol. 1, Elsevier, Amsterdam, The Netherlands, 1991.

[18] H. Takagi, Queueing Analysis-A Foundation of Performance Evaluation, vol. 3, Elsevier, Amsterdam, The Netherlands, 1993.

[19] N. Tian and Z. G. Zhang, Vacation Queueing Models-Theory and Applications, Springer, 2006.

[20] O. Berman, E. H. Kaplan, and D. G. Shimshak, "Deterministic approximations for inventory management at service facilities," IIE Transactions, vol. 25, no. 5, pp. 98-104, 1993.

[21] A. Krishnamoorthy and M. E. Islam, " $(s, S)$ inventory system with postponed demands," Stochastic Analysis and Applications, vol. 22, no. 3, pp. 827-842, 2004.

[22] B. Sivakumar and G. Arivarignan, "An inventory system with postponed demands," Stochastic Analysis and Applications, vol. 26, no. 1, pp. 84-97, 2008.

[23] P. Manuel, B. Sivakumar, and G. Arivarignan, "Perishable inventory system with postponed demands and negative customers," Journal of Applied Mathematics and Decision Sciences, vol. 2007, Article ID 94850, 12 pages, 2007.

[24] B. Sivakumar and G. Arivarignan, "A stochastic inventory system with postponed demands," Performance Evaluation, vol. 66, no. 1, pp. 47-58, 2009.

[25] D. P. Gaver, P. A. Jacobs, and G. Latouche, "Finite birthanddeath models in randomly changing environments," Advances in Applied Probability, vol. 16, pp. 715-731, 1984. 

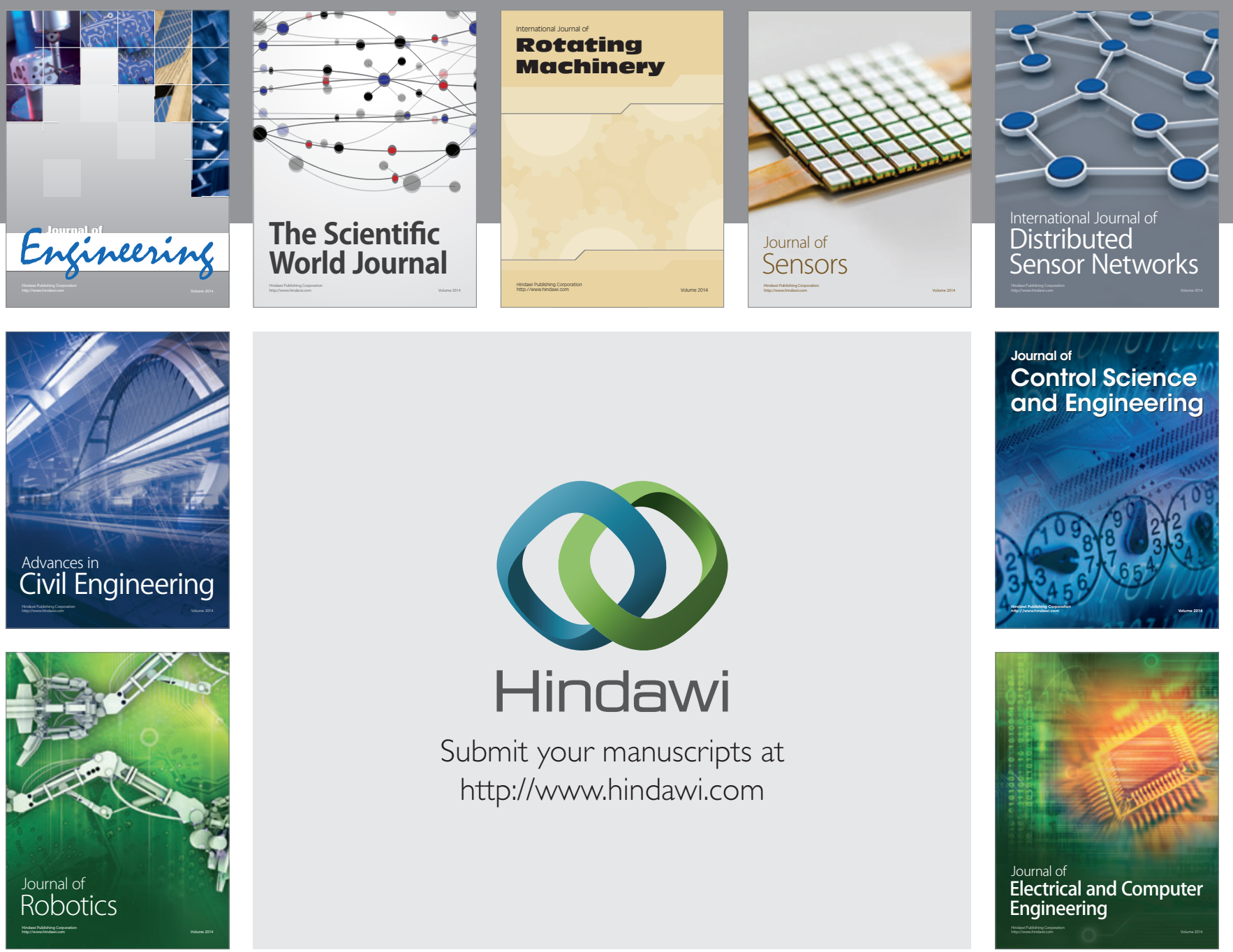

Submit your manuscripts at

http://www.hindawi.com
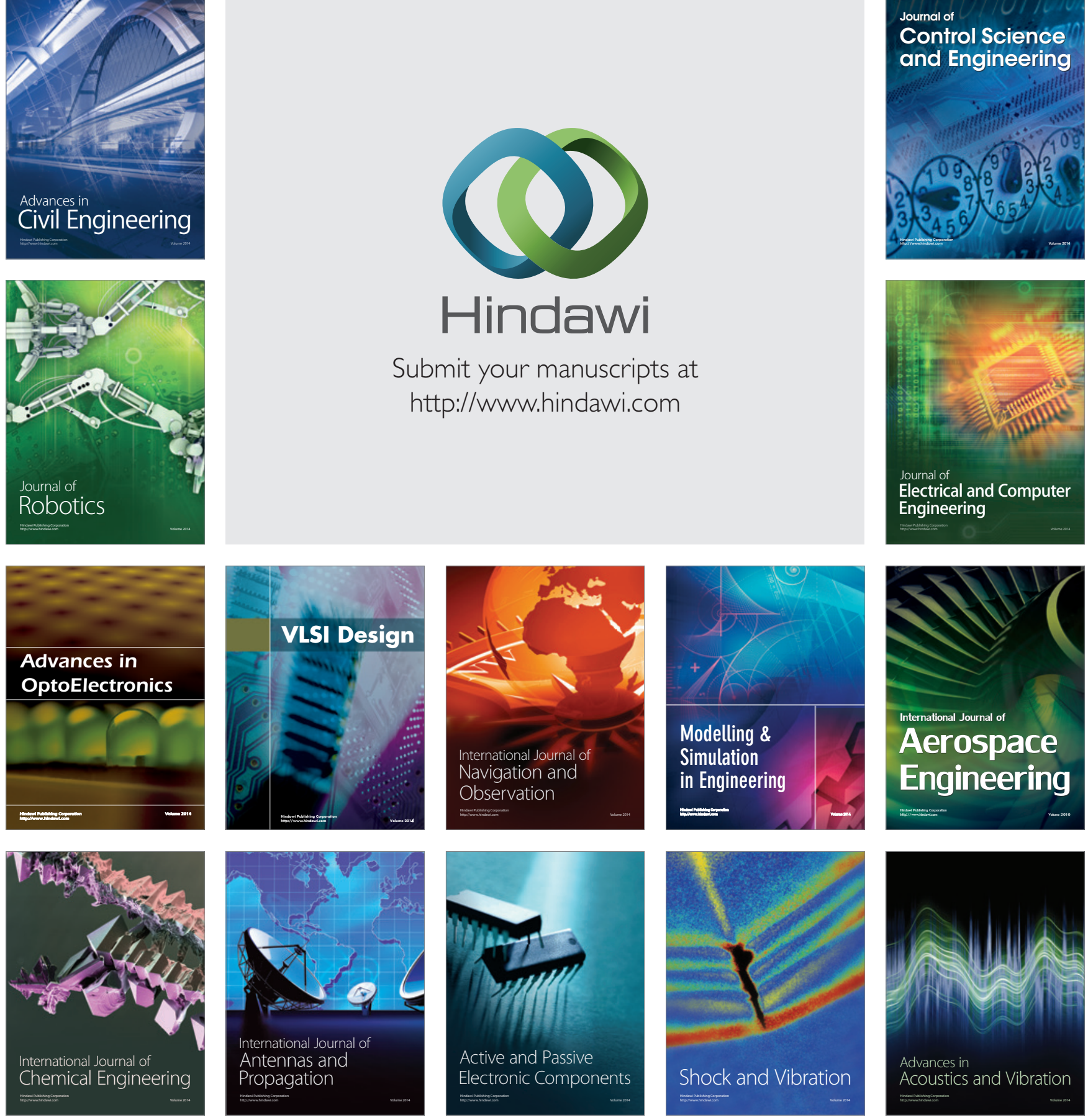